\title{
Entropy Viscosity Method for the Single Material Euler Equations in Lagrangian Frame th
}

\author{
Jean-Luc Guermond ${ }^{\mathrm{a}}$, Bojan Popov ${ }^{\mathrm{a}}$, Vladimir Tomov ${ }^{\mathrm{b}, *}$ \\ ${ }^{a}$ Department of Mathematics, Texas A\&M University 3368 TAMU, College Station, TX \\ $77843, U S A$. \\ ${ }^{b}$ Center for Applied Scientific Computing, Lawrence Livermore National Laboratory, P. O. \\ Box 808, L-561, Livermore, CA 94551, USA.
}

\begin{abstract}
A new finite element method for solving the Euler equations in Lagrangian coordinates is proposed. The method is stabilized by adding artificial diffusion terms compatible with positivity of mass and internal energy, a minimum principle on the specific entropy, and all generalized entropy inequalities. Two options of first-order artificial diffusion are considered. One is in the spirit of the Eulerian based method Guermond et al. [23, 22, Zingan et al. 47] and the other is similar to existing viscosity stabilizations in Lagrangian frame, e.g., Dobrev et al. [16]. The method is verified to be high-order for smooth solutions even with active viscosity terms. This is achieved by using high-order finite element spaces and an entropy-based viscosity stabilization that degenerates the first-order viscous terms. This stabilization automatically distinguishes smooth and singular regions. The formal accuracy and convergence properties of the proposed methods are tested on a series of benchmark problems. This is the first result extending the entropy-viscosity methodology to the Lagrangian hydrodynamics.
\end{abstract}

Keywords: Conservation equations, Lagrangian hydrodynamics, parabolic regularization, entropy viscosity, finite element method.

\section{Introduction}

We are interested in the present paper in simulating the 2D and 3D compressible Euler equations by using a Lagrangian method, i.e., one where the computational mesh moves with the fluid. These methods preserve material interface very well, thus they are the preferred approach for solving problems

\footnotetext{
This material is based upon work supported in part by the National Science Foundation grants DMS-1015984 and DMS-1217262, by the Air Force Office of Scientific Research, USAF, under grant/contract number FA99550-12-0358.

${ }^{*}$ Corresponding author, tomov2@llnl.gov
} 
dealing with compressible multi-phase and multi-material flows, for example numerical simulations of inertial confinement fusion (ICF) and impact problems. We refer the readers to Benson [3] for a review on hydrodynamics methods and detailed comparisons between Lagrangian and Eulerian methods.

There are roughly three major approaches for solving the Lagrangian hydrodynamics equations: staggered grid hydrodynamics (SGH); cell-centered hydrodynamics (CCH); finite element methods (FEM). SGH schemes represent the thermodynamic variables (density, energy, pressure) as constants over the mesh cells, and the kinematic variables (position, velocity) are evolved at the mesh nodes; see Caramana et al. [7, Burton [6, 5], Wilkins [46. CCH methods define all the flow variables as cell-averages quantities and nodal velocities are reconstructed by using approximate Riemann solvers; see Claisse et al. 11, Carré et al. [10, Maire [32, Maire and Breil [33, Maire et al. 34, Després and Mazeran [12. Approaches that combine both SGH and CCH concepts are derived in Loubère et al. 31, Maire et al. 35.

The purpose of the present paper is to further explore the FEM route. The use of FEM in Lagrangian hydrodynamics has recently re-gained interest after the work of Dobrev et al. [16, where the authors have shown that high-order FEM have excellent properties: geometry representation, symmetry preservation, resolution of shock fronts within one computational cell, high-order convergence for smooth solutions. The FEM formalism has been combined with SGH methods in Barlow [1, Scovazzi [38, Scovazzi et al. 39] and with $\mathrm{CCH}$ methods in Vilar et al. 44 in the form of a Discontinuous Galerkin scheme. Another approach that uses curvilinear NURBS (Non-Uniform Rational B-splines) meshes in order to obtain symmetry preservation can be found in Bazilevs et al. [2].

Since hydrodynamics calculations solve problems containing shock waves, special treatment is required in order to avoid post-shock oscillations. One way to resolve this issue is the artificial viscosity approach as originally suggested by Von Neumann and Richtmyer [45. When combined with FEM, this regularization is uniform with respect to the space dimension, the mesh type and the polynomial degree. High-order convergence can be achieved as long as the added diffusion terms are active only in the singular regions of the solution. Further requirements and specific artificial viscosity terms for the Lagrangian case have been proposed by Caramana et al. 8, Caramana and Loubère [9], Shashkov and Campbell [42, Kolev and Rieben [26, Lipnikov and Shashkov 30.

In this paper we propose a new artificial-viscosity-based high-order finite element method for solving the Euler equations of the compressible gas dynamics in Lagrangian coordinates. We generalize to the Lagrangian framework the ideas presented in Guermond [19, Guermond et al. 23], Guermond and Popov [20] and derive a regularized Lagrangian system that is compatible with all the generalized entropy inequalities, see Harten et al. [25, Guermond and Popov [20], and the minimum principle on the specific entropy, see Tadmor [43. In addition to the standard viscous regularization of the momentum equation, artificial diffusion is added to both the mass and the energy equations, thereby 
allowing a control of density and energy oscillations near shocks and contact regions. Note that although the mesh moves with the fluid velocity, adding mass viscosity allows material to flow through the mesh and this might create technical difficulties when dealing with multi-material flow problems. This issue is not addressed here since we are only concerned in this paper with single phase flows. This approach is nonetheless useful in non-ideal gas simulations where contacts transform into composite waves. The amount of applied nonlinear dissipation is based on the local residual of the entropy equation; see Lax [29]. One immediate consequence of this choice is that the method automatically distinguishes between smooth and singular regions. The proposed method combines the following features: (i) the equations are regularized in a way that provides control over oscillations around contact discontinuities as well as oscillations in shock regions. (ii) the method produces high-order convergence rates for smooth solutions even with active viscosity terms. This is achieved by using high-order finite element spaces and, more importantly, an entropybased viscosity that automatically distinguishes between smooth and singular regions; (iii) the proposed diffusion terms are in agreement with the general requirements stated by Kolev and Rieben [26] for artificial tensor viscosities. This work is motivated and influenced by the idea of entropy-production-based artificial viscosity which was developed in Guermond et al. [23, 22, Zingan et al. [47] in the Eulerian frame of reference, and the application of high-order finite elements in Lagrangian Hydrodynamics presented in Dobrev et al. [16. The finite element framework is similar to that in Dobrev et al. [16, however, our approach is based on different viscous regularizations, viscosity coefficients, and finite element spaces.

This paper is organized as follows: in Section 2, we discuss the basic entropy principles that motivate the specific form of the viscous regularization, and derive the continuous form of the regularized system in the Lagrangian frame of reference. In Section 3, we describe the finite element discretization and the viscosity coefficients. Numerical test on various model Lagrangian problems are presented in Section 4 .

\section{Preliminaries}

In this section we state the problem and give the theoretical background that motivates the particular form of the viscous regularization that is chosen. We formulate the Lagrangian system of equations that serves as a starting point for the finite element discretization.

We start with the compressible Euler equations in Eulerian coordinates:

$$
\begin{aligned}
\frac{\partial}{\partial t} \rho+\nabla \cdot(\boldsymbol{u} \rho) & =0, \\
\frac{\partial}{\partial t}(\rho \boldsymbol{u})+\nabla \cdot(\rho \boldsymbol{u} \otimes \boldsymbol{u})+\nabla p & =0, \\
\frac{\partial}{\partial t}(\rho E)+\nabla \cdot(\boldsymbol{u} \rho E+\boldsymbol{u} p) & =0,
\end{aligned}
$$


where the dependent variables are the density $\rho$, the momentum $\left(\rho u_{1} \ldots \rho u_{d}\right)^{T}$ and the total energy $\rho E$. We use the usual convention for vector and tensor operations: for column vectors $\boldsymbol{a}=\left(a_{1} \ldots a_{d}\right)^{T}, \boldsymbol{b}=\left(b_{1} \ldots b_{d}\right)^{T}$ and order 2 tensors $\mathrm{g}$, h with entries $g_{i j}, h_{i j}$ where $i, j=1 \ldots d$, we have:

$$
\begin{array}{r}
\boldsymbol{a} \otimes \boldsymbol{b}=\boldsymbol{a b}^{T}, \quad(\nabla \boldsymbol{a})_{i j}=\frac{\partial a_{j}}{\partial x_{i}}, \quad(\nabla \cdot \mathrm{g})_{i}=\sum_{j=1}^{d} \frac{\partial g_{j i}}{\partial x_{j}}, \\
(\boldsymbol{a} \cdot \mathrm{g})_{i}=\sum_{j=1}^{d} a_{j} g_{j i}, \quad(\mathrm{~g} \cdot \boldsymbol{a})_{i}=\sum_{j=1}^{d} g_{i j} a_{j}, \quad \mathrm{~g}: \mathrm{h}=\sum_{i j=1}^{d} g_{i j} h_{i j} .
\end{array}
$$

The Euclidian norm in $\mathbb{R}^{d}$ and $\mathbb{R}^{d \times d}$ is denoted $\|\cdot\|_{\ell^{2}}$ indifferently. The following standard identities are going to be used throughout this document:

$$
\begin{aligned}
& \nabla \cdot(\boldsymbol{a} \otimes \boldsymbol{b})=\boldsymbol{a} \cdot \nabla \boldsymbol{b}+\boldsymbol{b} \nabla \cdot \boldsymbol{a}, \quad \nabla \cdot(\mathrm{g} \cdot \boldsymbol{a})=\mathrm{g}: \nabla \boldsymbol{a}+(\nabla \cdot \mathrm{g}) \cdot \boldsymbol{a}, \\
& {\left[\nabla\left(\frac{\boldsymbol{a}^{2}}{2}\right)\right] \cdot \boldsymbol{b}=\boldsymbol{a} \cdot(\boldsymbol{b} \cdot \nabla \boldsymbol{a}) .}
\end{aligned}
$$

We focus our attention to the equation of state of an ideal gas, although most of the arguments in the present paper are independent of the exact nature of the equation of state. The pressure $p$ is given by

$$
p=(\gamma-1) \rho e,
$$

where $\gamma$ is the ratio of constant pressure and constant volume heat capacities. Instead of using the conservation equation for the total energy (3), a common practice in Lagrangian gasdynamics consists of working with the balance equation for the internal energy:

$$
\frac{\partial}{\partial t}(\rho e)+\nabla \cdot(\boldsymbol{u} \rho e)+p \nabla \cdot \boldsymbol{u}=0
$$

Throughout this document we also use the vector form of the system (1)-(3) to simplify the notation:

$$
\frac{\partial \boldsymbol{v}}{\partial t}+\sum_{m=1}^{d} \frac{\partial \boldsymbol{f}^{\boldsymbol{m}}(\boldsymbol{v})}{\partial x_{m}}=0,
$$

where $\boldsymbol{v}=\left(\rho, \rho u_{1}, \ldots, \rho u_{d}, \rho E\right)^{T}$, and $\boldsymbol{f}=\left(\boldsymbol{f}^{\mathbf{1}} \ldots \boldsymbol{f}^{\boldsymbol{d}}\right)^{T}$ are the fluxes for each dimension.

\subsection{Entropy principles}

Due to the nonlinearities in (6), solutions that are initially smooth may become discontinuous within finite time. Hence, solutions are defined globally in time by considering the equations in the distributional sense. However, the resulting weak problem may admit infinitely many weak solutions, and a selection mechanism for determining whether a weak solution is physical or not must be 
invoked. A weak solution $\boldsymbol{v}$ of $(6)$ is called admissible in the vanishing viscosity sense if it is the limit of solutions $\boldsymbol{v}^{\delta}$ in $L_{x, t, \text { loc }}^{1}$ as $\delta \rightarrow 0$ where $\boldsymbol{v}^{\delta}$ are the solutions of the viscous equation

$$
\frac{\partial \boldsymbol{v}^{\delta}}{\partial t}+\nabla \cdot \boldsymbol{f}\left(\boldsymbol{v}^{\delta}\right)=\delta \Delta \boldsymbol{v}^{\delta}, \quad \boldsymbol{v}^{\delta}(\cdot, 0)=\boldsymbol{v}^{0}
$$

If a sequence of solutions of $(7)$ can be proved to converge in $L_{x, t, \text { loc }}^{1}$, then one can show that the limit is a weak solution. While the convergence has been established in the scalar case and for some specific systems, see Krushkov [27, Lax [28, Ding et al. [13, DiPerna [14, 15], the result for the case of the Euler equations is still an open problem. In the rest of this paper, we assume that the solutions of (7) converges as $\delta \rightarrow 0$ and we use the viscous limit as the criterion for selecting a "physical" weak solution. This result is known to hold for any hyperbolic systems in one space dimensions with small initial data, see Bianchini and Bressan (4) for details. With this assumption, equation (7) is used to deduce other conditions which are easier to verify numerically. This is done through a function called "entropy", which is a generalization of the thermodynamical entropy.

Definition 1. A function $S(\boldsymbol{v})$ is an entropy function for the system (6), if:

1. There is vector-valued function $\boldsymbol{F}(\boldsymbol{v})$, called entropy flux, such that the following identity holds $\partial_{\boldsymbol{v}} S^{T}\left(\partial_{\boldsymbol{v}} \boldsymbol{f}^{m}\right)=\left(\partial_{\boldsymbol{v}} F^{m}\right)^{T}, m=1 \ldots d$,

2. $S$ is a convex function of $\boldsymbol{v}$.

Now we derive an entropy $S$ that is compatible with Definition 1. Note that the first condition in the above definition implies (after taking a dot product of (6) with $\partial_{v} S$ ) that:

$$
\frac{\partial S(\boldsymbol{v})}{\partial t}+\nabla \cdot \boldsymbol{F}(\boldsymbol{v})=0
$$

for every smooth solution $\boldsymbol{v}$.

We follow the approach first introduced by Harten [24]. For the EOS $p=$ $(\gamma-1) \rho e$ and $T=(\gamma-1) e$, we define the quantity

$$
s(\rho, e):=\log \left(p \rho^{-\gamma}\right)=\log \left((\gamma-1) e \rho^{1-\gamma}\right)=\log ((\gamma-1) e)+(1-\gamma) \log \rho,
$$

which we call "specific entropy". Notice that this is not exactly the physical specific entropy (it doesn't satisfy $T d s=d e-\frac{p}{\rho^{2}} d \rho$ ). Its derivatives with respect to density and energy are

$$
\frac{\partial s}{\partial \rho}=\frac{1-\gamma}{\rho}, \quad \frac{\partial s}{\partial e}=\frac{1}{e} .
$$

For smooth solutions the specific entropy $s$ satisfies $\frac{\partial s(\boldsymbol{v})}{\partial t}+\boldsymbol{u} \cdot \nabla s(\boldsymbol{v})=0$. Note that we abuse the notation by using indifferently $s(\boldsymbol{v})$ or $s(\rho, e)$. For any scalar differentiable function $f(s)$, the conservation of mass implies that

$$
\frac{\partial}{\partial t}(\rho f(s))+\nabla \cdot(\boldsymbol{u} \rho f(s))=0 .
$$


We set

$$
S:=-\rho f(s), \quad \boldsymbol{F}:=-\boldsymbol{u} \rho f(s)
$$

and arrive at the entropy equation (8). It is shown in Harten et al. 25] that the function $S$ is a convex function of the conservative variables if and only if

$$
f^{\prime}(s)>0, \quad f^{\prime}(s) \frac{\gamma-1}{\gamma}+f^{\prime \prime}(s)>0 .
$$

A family of functionals that satisfies 13 is

$$
f(s)=\frac{\gamma+\alpha}{\gamma-1} \exp \left(\frac{s}{\gamma+\alpha}\right) \Rightarrow f^{\prime}(s)=\frac{1}{\rho(\gamma-1)}\left(p \rho^{\alpha}\right)^{\frac{1}{\gamma+\alpha}},
$$

where $\alpha>0$ is some constant. Then we have the following family of entropies:

$$
S=-\rho f(s)=\frac{\gamma+\alpha}{1-\gamma}\left(p \rho^{\alpha}\right)^{\frac{1}{\gamma+\alpha}},
$$

so that $S$ satisfies both conditions of Definition 1 for any $\alpha>0$.

Based on the results in Harten et al. 25], Tadmor [43, the following "physical" properties are satisfied by strong viscosity limits:

- Positivity of density and internal energy.

- Entropy inequality. A weak solution of (6) is entropy-admissible if it satisfies the inequality

$$
\frac{\partial S(\boldsymbol{v})}{\partial t}+\nabla \cdot \boldsymbol{F}(\boldsymbol{v}) \leq 0
$$

in the distribution sense for every pair of entropy pair $(S, \boldsymbol{F})$.

- Minimum principle on the specific entropy, derived by Tadmor 43]: if $\boldsymbol{v}(\boldsymbol{x}, t)$ is a weak solution of (6) that satisfies the entropy inequality (15), then for the specific entropy $s$ defined in $(9)$ we have

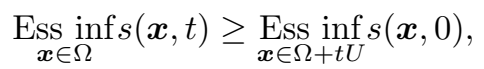

for all $t>0$ and any domain $\Omega$, where $U$ is the maximum of $\|\boldsymbol{u}\|_{\ell^{2}}$ in $\Omega$.

Remark 2.1. When there is a source $Q$ of internal energy, the entropy equation (11) becomes: $\frac{\partial}{\partial t}(\rho f(s))+\nabla \cdot(\rho f(s))-Q s_{e} f^{\prime}(s)=0$, i.e.,

$$
\frac{\partial S}{\partial t}+\nabla \cdot S+\frac{Q f^{\prime}(s)}{e}=0 .
$$

Remark 2.2. The amount of violation of 111 or $(17)$ is called "entropy production". It can be non-zero only in regions of discontinuities of the solution. We will use this quantity to scale the artificial viscosity terms, i.e., it will be our shock detector (and hence the name "Entropy Viscosity method"). 
Remark 2.3. We usually choose $\alpha=1$ in the definition of $S$ in (14). For smaller values of $\alpha$ the explicit density dependence decreases, for example $\alpha=0$ would imply that $S$ is smooth in contact regions (since the pressure is continuous across contact discontinuities). In that case the entropy production there is zero and the corresponding shock detector would not add viscosity in contacts. The key reason for this behavior is that the function $S$ is not strictly convex when $\alpha=0$.

Remark 2.4. We have a minus sign in the definitions of $S$ and $\boldsymbol{F}$, because we want to be consistent with (13). The specific entropy used by Harten et al. [25] is that in (9) multiplied by a negative constant, namely it is $\log \left(\rho e^{1 /(1-\gamma)}\right)$ (which is minus the physical specific entropy).

\subsection{Viscous regularization}

Artificial viscous terms must be introduced to be consistent with entropy dissipation, see Guermond and Popov [20], and to preserve invariant domains, see Frid [17, Guermond and Popov [21]. A common way to regularize (1)-(3) consists of adding diffusion terms that are similar to the viscosity and thermal diffusion in the Navier-Stokes equations. This approach, however, is in agreement with the minimum principle of the specific entropy (16) only if the thermal diffusion is zero, see Theorem 8.2.3 in Serre [41]. If the thermal diffusion is absent, there is no mechanism to control contact discontinuities, since in contact regions the velocity is constant (i.e., there is no compression) and the viscosity is inactive since the gradient of the velocity is zero across the contact. In the Eulerian frame, one would see uncontrolled oscillations resulting from the Gibbs effect as these contact discontinuities move through the computational mesh. This problem can be concealed in Lagrangian methods by aligning the initial contact discontinuities with the cell boundaries and by using discontinuous spaces, but the above problem nevertheless re-appears if contact lines or surfaces form in time.

Our goal is to satisfy the entropy inequality (15) and minimum principle on the specific entropy (16). It is shown in Guermond and Popov [20] that to satisfy both these requirement, one needs to add mass and thermal viscosity, in addition to the standard momentum viscosity. This makes the method more diffusive than those using the Navier-Stokes regularization approach only, but it is essential for removing oscillation both in contact and shock regions. A regularization that takes into account all the above considerations is described in [20] and has the form

$$
\begin{array}{r}
\frac{\partial \rho}{\partial t}+\nabla \cdot(\rho \boldsymbol{u})=\nabla \cdot \boldsymbol{w}, \\
\frac{\partial}{\partial t}(\rho \boldsymbol{u})+\nabla \cdot(\rho \boldsymbol{u} \otimes \boldsymbol{u})+\nabla p=\nabla \cdot \mathrm{g}, \\
\frac{\partial}{\partial t}(\rho E)+\nabla \cdot(\rho E \boldsymbol{u}+p \boldsymbol{u})=\nabla \cdot(\boldsymbol{h}+\mathrm{g} \cdot \boldsymbol{u}),
\end{array}
$$


where the viscous terms are:

$$
\boldsymbol{w}=\lambda \nabla \rho, \quad \mathrm{g}=\nu \rho \nabla \boldsymbol{u}+\boldsymbol{w} \otimes \boldsymbol{u}, \quad \boldsymbol{h}=\lambda \nabla(\rho e)-\frac{\boldsymbol{u}^{2}}{2} \boldsymbol{w} .
$$

Here $\lambda$ and $\nu$ are coefficients that control the amount of added diffusion. These coefficients must have units of (speed $\times$ distance).

\subsection{Lagrangian formulation}

We want to solve the system (18)-20 in the Lagrangian frame of reference. We can think of the medium as a set of particles having original positions $\boldsymbol{x}_{0}$. In the Lagrangian setting these particles move with the fluid velocity, namely

$$
\frac{d}{d t} \boldsymbol{x}\left(\boldsymbol{x}_{0}, t\right):=\boldsymbol{u}(\boldsymbol{x}, t)
$$

Then the material derivative (also called total, Lagrangian, convective, etc.) of a scalar- or vector-valued function $\beta=\beta\left(\boldsymbol{x}\left(\boldsymbol{x}_{0}, t\right), t\right)$ is

$$
\frac{d}{d t} \beta\left(\boldsymbol{x}\left(\boldsymbol{x}_{0}, t\right), t\right)=\frac{\partial \beta(\boldsymbol{x}, t)}{\partial t}+\boldsymbol{u}(\boldsymbol{x}, t) \cdot \nabla_{\boldsymbol{x}} \beta(\boldsymbol{x}, t) .
$$

Now we rewrite the equations 18 - 20 by using the total derivatives. The conservation of mass equation (18) becomes

$$
\frac{d \rho}{d t}=-\rho \nabla \cdot \boldsymbol{u}+\nabla \cdot(\lambda \nabla \rho)
$$

The velocity equation 19 becomes

$$
\rho \frac{d \boldsymbol{u}}{d t}=-\nabla p+\nabla \cdot(\nu \rho \nabla \boldsymbol{u})+(\lambda \nabla \rho) \cdot(\nabla \boldsymbol{u}) .
$$

The equation for total energy 20 becomes

$$
\rho \frac{d E}{d t}=-\nabla \cdot(p \boldsymbol{u})+\nabla \cdot(\lambda \rho \nabla e+\nu \rho \nabla \boldsymbol{u} \cdot \boldsymbol{u})+(\lambda \nabla \rho) \cdot \nabla E .
$$

Working with the equation for the internal energy instead of 22 is more common in Lagrangian formulation. Taking the dot product of (21) with $\boldsymbol{u}$ and subtracting the result from 22 we derive

$$
\rho \frac{d e}{d t}=-p \nabla \cdot \boldsymbol{u}+\nabla \cdot(\lambda \rho \nabla e)+\nu \rho \nabla \boldsymbol{u}: \nabla \boldsymbol{u}+(\nabla e) \cdot(\lambda \nabla \rho) .
$$

Then the final Lagrangian system that we propose is:

$$
\begin{aligned}
& \frac{d}{d t} \boldsymbol{x}\left(\boldsymbol{x}_{0}, t\right)=\boldsymbol{u}(\boldsymbol{x}, t), \\
& \frac{d \rho}{d t}=-\rho \nabla \cdot \boldsymbol{u}+\nabla \cdot(\lambda \nabla \rho), \\
& \rho \frac{d \boldsymbol{u}}{d t}=-\nabla p+\nabla \cdot(\nu \rho \nabla \boldsymbol{u})+(\lambda \nabla \rho) \cdot(\nabla \boldsymbol{u}), \\
& \rho \frac{d e}{d t}=-p \nabla \cdot \boldsymbol{u}+\nabla \cdot(\lambda \rho \nabla e)+\nu \rho \nabla \boldsymbol{u}: \nabla \boldsymbol{u}+(\nabla e) \cdot(\lambda \nabla \rho) .
\end{aligned}
$$


The dependent variables are the position $\boldsymbol{x}\left(\boldsymbol{x}_{0}, t\right)$, the density $\rho(\boldsymbol{x}, t)$, velocity $\boldsymbol{u}(\boldsymbol{x}, t)$, the specific internal energy $e(\boldsymbol{x}, t)$. We recall that the equation of state (4) is $p=(\gamma-1) \rho e$. Note that the system (24)-(25)-(26) is written in non-conservative form, which is standard in the Lagrangian hydrodynamics literature. , and as a result conservation of the total mass, momentum and total energy is not guaranteed a priori. Our numerical experiments have shown that this has never been an issue.

\section{Discretization details}

In this subsection we derive a fully-discrete finite element method for the system (23)-(26). We propose a semi-discrete form with high-order continuous finite element spaces and discuss the notions of mesh representation, mappings, length scale, viscosity coefficients, and time discretization.

\subsection{Notation}

Let $\Omega_{0}$ be the domain at time 0 and $\Omega(t)$ be the domain at time $t$. Then $\left\{\mathcal{K}_{h}\right\}_{h>0}$ is a mesh sequence with no hanging nodes that discretizes $\Omega_{0}$. As we progress in time, the initial mesh is deformed by moving the Lagrange nodes of the mesh according to the rule (23) where the $\boldsymbol{u}$ is made explicit in time. The reference cell is denoted $\widehat{K}$. Given a cell $K_{0} \in \mathcal{K}_{h}$, we denote by $\Phi_{K_{0}}: \widehat{K} \rightarrow K_{0}$ the geometric transformation. As time increases, $K_{0}$ is deformed into $K$ and we denote $\Phi_{K}: \widehat{K} \rightarrow K$ the corresponding geometric transformation; details on how these transformations are constructed are given in 3.3 . Note that $\Phi_{K}$ and $K$ are time-dependent, but we henceforth abuse the notation by omitting the time index. A natural mapping from $K_{0}$ to $K$ is given by $\Phi_{K} \circ \Phi_{K_{0}}^{-1}: K_{0} \rightarrow K$; note that $\Phi_{K} \circ \Phi_{K_{0}}^{-1}$ is the identity at $t=0$. To simplify the notation we introduce $\boldsymbol{x}:=\Phi_{K}(\widehat{\boldsymbol{x}})$ and $\boldsymbol{x}_{0}:=\Phi_{K_{0}}(\widehat{\boldsymbol{x}})$ for any $\widehat{\boldsymbol{x}} \in \widehat{K}$.

We introduce a scalar-valued nodal finite element space defined with respect to the initial mesh $K_{0}$ as follows:

$$
Q_{k}=\left\{v \in C^{0}\left(\Omega_{0}\right) ;\left.v\right|_{K_{0}} \circ \Phi_{K_{0}} \in \mathbb{Q}_{k}, \forall K_{0} \in \mathcal{K}_{h}\right\} .
$$

where $\mathbb{Q}_{k}$ is the set of multivariate polynomials of partial degree at most $k$. Let $\left\{\boldsymbol{a}_{1}, \ldots, \boldsymbol{a}_{N}\right\}$ be the Lagrange nodes of the mesh and let $\left\{\varphi_{1}, \ldots, \varphi_{N}\right\}$ be the associated nodal shape functions, i.e., $\varphi_{i}\left(\boldsymbol{a}_{j}\right)=\delta_{i j}$. Since the mesh nodes move, we define the time-dependent basis functions by

$$
\left.\varphi(\boldsymbol{x}, t):=\varphi\left(\boldsymbol{x}_{0}\right) \quad \text { (equivalent to } \varphi(\boldsymbol{x}, 0):=\varphi\left(\boldsymbol{x}_{0}\right), \frac{d}{d t} \varphi(\boldsymbol{x}, t):=0\right),
$$

where by convention $\boldsymbol{x}=\Phi_{K} \circ \Phi_{K_{0}}^{-1}\left(\boldsymbol{x}_{0}\right)$. Note that here again we abuse the notation by using the same symbol for the reference shape function and the transformed shape function. Examples of $Q_{4}$ basis functions on the original and transformed meshes are shown in Figure $1 \mathrm{a}$ and Figure 1b, Note that approximation using such functions stays $H^{1}$-conforming in time. Shape functions on the reference cell are denoted by $\widehat{\varphi}_{i}(\widehat{\boldsymbol{x}}), i=1 \ldots \widehat{N}$. 
From this point forward, by $\rho, \boldsymbol{u}, e, \boldsymbol{x}, p, S$ we refer to the discrete versions of the dependent variables in $Q_{k}$. Quantities that only depend on $\boldsymbol{x}_{0}$ are defined at the initial time, for example $\rho\left(\boldsymbol{x}_{0}\right)$ is the approximation of the initial density.

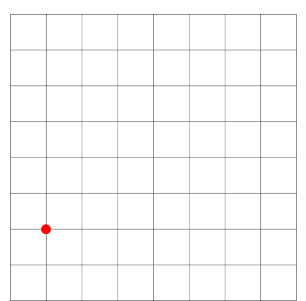

(a) Original mesh and $Q_{4}$ shape function.

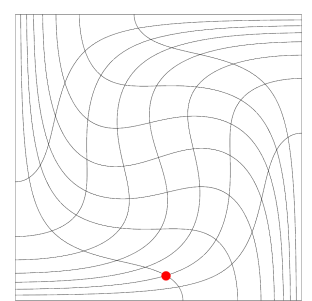

(b) Transformed mesh using the TaylorGreen vortex motion and $Q_{4}$ shape function.

Figure 1: Example of a $Q_{4}$ shape function on the original mesh and transformed mesh.

Remark 3.1. The methodology presented in this paper works in principle for any type of continuous finite elements (it is not restricted to quadrangular and hexahedral meshes) but in this paper we only consider $Q_{k}$ approximations in the implementation of the method.

\subsection{Semi-discrete form}

We obtain a semi-discrete form of the problem by multiplying every equation in (24)- 26 with a test function and integrating over $\Omega(t)$. We use spaces of same polynomial degree for all the dependent variables, namely we seek $(\rho, \boldsymbol{u}, e, \boldsymbol{x}) \in$ $\left(Q_{k} \times Q_{k}^{d} \times Q_{k} \times Q_{k}^{d}\right)$. Our tests did not show any significant benefits in using different polynomial degrees for the kinematic and thermodynamic spaces.

- Density - for every $j=1 \ldots N$ we have

$$
\begin{aligned}
\int_{\Omega(t)} \frac{d \rho(\boldsymbol{x}, t)}{d t} \varphi_{j}(\boldsymbol{x}, t) \mathrm{d} \boldsymbol{x}= & -\int_{\Omega(t)} \rho(\boldsymbol{x}, t) \nabla \cdot \boldsymbol{u}(\boldsymbol{x}, t) \varphi_{j}(\boldsymbol{x}, t) \mathrm{d} \boldsymbol{x} \\
& -\int_{\Omega(t)} \lambda \nabla \rho(\boldsymbol{x}, t) \cdot \nabla \varphi_{j}(\boldsymbol{x}, t) \mathrm{d} \boldsymbol{x} .
\end{aligned}
$$

- Velocity - for every dimension $m=1 \ldots d$ and every $j=1 \ldots N$ we have

$$
\begin{aligned}
& \int_{\Omega(t)} \rho(\boldsymbol{x}, t) \frac{d u_{m}(\boldsymbol{x}, t)}{d t} \varphi_{j}(\boldsymbol{x}, t) \mathrm{d} \boldsymbol{x}=-\int_{\Omega(t)} \frac{\partial p(\boldsymbol{x}, t)}{\partial x_{m}} \varphi_{j}(\boldsymbol{x}, t) \mathrm{d} \boldsymbol{x} \\
& -\int_{\Omega(t)} \nu \rho \nabla u_{m}(\boldsymbol{x}, t) \cdot \nabla \varphi_{j}(\boldsymbol{x}, t) \mathrm{d} \boldsymbol{x}+\int_{\Omega(t)} \lambda \nabla u_{m}(\boldsymbol{x}, t) \cdot \nabla \rho(\boldsymbol{x}, t) \varphi_{j}(\boldsymbol{x}, t) \mathrm{d} \boldsymbol{x} .
\end{aligned}
$$


- Internal energy - for every $j=1 \ldots N$ we have

$$
\begin{aligned}
& \int_{\Omega(t)} \rho(\boldsymbol{x}, t) \frac{d e(\boldsymbol{x}, t)}{d t} \varphi_{j}(\boldsymbol{x}, t) \mathrm{d} \boldsymbol{x}=-\int_{\Omega(t)} p(\boldsymbol{x}, t) \nabla \cdot \boldsymbol{u}(\boldsymbol{x}, t) \varphi_{j}(\boldsymbol{x}, t) \mathrm{d} \boldsymbol{x} \\
& -\int_{\Omega(t)} \lambda \rho(\boldsymbol{x}, t) \nabla e(\boldsymbol{x}, t) \cdot \nabla \varphi_{j}(\boldsymbol{x}, t) \mathrm{d} \boldsymbol{x}+\int_{\Omega(t)} \lambda \nabla e(\boldsymbol{x}, t) \cdot \nabla \rho(\boldsymbol{x}, t) \varphi_{j}(\boldsymbol{x}, t) \mathrm{d} \boldsymbol{x} \\
& +\int_{\Omega(t)} \nu \rho(\boldsymbol{x}, t) \nabla \boldsymbol{u}(\boldsymbol{x}, t): \nabla \boldsymbol{u}(\boldsymbol{x}, t) \varphi_{j}(\boldsymbol{x}, t) \mathrm{d} \boldsymbol{x} .
\end{aligned}
$$

And the position function $\boldsymbol{x}\left(\boldsymbol{x}_{0}, t\right)$ is simply evolved by solving $\frac{d}{d t} \boldsymbol{x}\left(\boldsymbol{x}_{0}, t\right)=$ $\boldsymbol{u}(\boldsymbol{x}, t)$ with the initial condition $\boldsymbol{x}\left(\boldsymbol{x}_{0}, 0\right)=\boldsymbol{x}_{0}$ using the chosen time integrator.

Remark 3.2. One drawback of adding mass viscosity is that the resulting mass matrices are time-dependent. For example, the left-hand side of (30) and the corresponding matrix are

$$
\begin{gathered}
\int_{\Omega(t)} \rho(\boldsymbol{x}, t) \frac{d}{d t}\left(\sum_{i=1}^{N} e_{i}(t) \varphi_{i}(\boldsymbol{x}, t)\right) \varphi_{j}(\boldsymbol{x}, t) \mathrm{d} \boldsymbol{x}=\sum_{i=1}^{N} \frac{d e_{i}(t)}{d t} \int_{\Omega(t)} \rho(\boldsymbol{x}, t) \varphi_{i}(\boldsymbol{x}, t) \varphi_{j}(\boldsymbol{x}, t) \mathrm{d} \boldsymbol{x}, \\
\left(M_{e}\right)_{i j}=\int_{\Omega_{0}} \rho\left(\boldsymbol{x}\left(\boldsymbol{x}_{0}, t\right), t\right) \varphi_{i}\left(\boldsymbol{x}_{0}\right) \varphi_{j}\left(\boldsymbol{x}_{0}\right)\left|\operatorname{det} J_{\boldsymbol{x}_{0} \rightarrow \boldsymbol{x}}\right| \mathrm{d} \boldsymbol{x}_{0} .
\end{gathered}
$$

It is not true here that $\rho\left(\boldsymbol{x}\left(\boldsymbol{x}_{0}, t\right), t\right)\left|\operatorname{det} J_{\boldsymbol{x}_{0} \rightarrow \boldsymbol{x}}\right|=\rho\left(\boldsymbol{x}_{0}\right)$, which would otherwise be the case if we did not have the additional viscous term in the mass equation, see Dobrev et al. [16].

Remark 3.3. Note that no special effort is made here to make the method exactly conservative. Actually the method, semi-discretized in space as explained above but with continuous time, is conservative. More precisely, summing 28 over $j$ and using the partition of unity property, we have $\int_{\Omega(t)} \frac{d \rho(\boldsymbol{x}, t)}{d t} \mathrm{~d} \boldsymbol{x}+$ $\int_{\Omega(t)} \rho(\boldsymbol{x}, t) \nabla \cdot \boldsymbol{u}(\boldsymbol{x}, t) \mathrm{d} \boldsymbol{x}=0$, which, owing to Reynolds Theorem, gives that the time derivative of $\int_{\Omega(t)} \rho(\boldsymbol{x}, t) \mathrm{d} \boldsymbol{x}$ is zero; whence conservation of mass. This property does not hold though when time discretization is applied; strictly speaking the fully discrete algorithm as described in $\$ 3.7$ is not conservative.

\subsection{Mesh representation and mappings}

Assuming again that the velocity field $\boldsymbol{u}(\boldsymbol{x}, t)$ is known, the motion of the mesh is controlled by the motion of the nodes $\left\{\boldsymbol{a}_{1} \ldots \boldsymbol{a}_{N}\right\}$, i.e., we define the functions $\left\{\boldsymbol{X}_{1}(t) \ldots \boldsymbol{X}_{N}(t)\right\}$ by

$$
\frac{\mathrm{d} \boldsymbol{X}_{i}(t)}{\mathrm{d} t}=\boldsymbol{u}\left(\boldsymbol{X}_{i}(t), t\right), \quad \boldsymbol{X}_{i}(0)=\boldsymbol{a}_{i}
$$


Then the trajectory of a particle with original position $\boldsymbol{x}_{0}$ is obtained by using the finite element expansion

$$
\boldsymbol{x}\left(\boldsymbol{x}_{0}, t\right)=\sum_{j=1}^{N} \boldsymbol{X}_{j}(t) \varphi_{j}\left(\boldsymbol{x}_{0}\right)
$$

The use of high-order polynomial basis functions implies that the node are connected by high-order curves and the mesh is curvilinear. Notice that in order to obtain the position of any point in the computational mesh, we only need the positions of the nodes. This approach is very efficient since it does not involve any complicated curve reconstructions.

Let $j: \mathcal{K}_{h} \times\{1 \ldots \widehat{N}\} \ni(K, i) \longmapsto j(K, i) \in\{1 \ldots N\}$ be the connectivity array associating the reference shape functions with the global shape functions over each cell, i.e., $\varphi_{j(K, i)}\left(\Phi_{K}(\widehat{\boldsymbol{x}})\right)=\widehat{\varphi}_{i}(\widehat{\boldsymbol{x}})$ for all $\widehat{\boldsymbol{x}} \in \widehat{K}$ for all $t \geq 0$. The formula (32) provides a straightforward way to define the time-dependent position mapping $\Phi_{K}(\cdot, t): \widehat{K} \ni \widehat{\boldsymbol{x}} \longmapsto \boldsymbol{x} \in K$ from the reference cell $\widehat{K}$ to an actual cell of interest $K$ :

$$
\Phi_{K}(\widehat{\boldsymbol{x}}, t):=\boldsymbol{x}(\widehat{\boldsymbol{x}}, t)=\sum_{i=1}^{\widehat{N}} \boldsymbol{X}_{j(K, i)}(t) \widehat{\varphi}_{i}(\widehat{\boldsymbol{x}}),
$$

where recall that $\widehat{\varphi}_{1} \ldots \widehat{\varphi}_{\widehat{N}}$ are the basis functions on the reference cell. The time-dependent Jacobian matrix of this mapping is then simply

$$
J(\widehat{\boldsymbol{x}}, t):=\frac{\partial \boldsymbol{x}}{\partial \widehat{\boldsymbol{x}}}=\sum_{i=1}^{\widehat{N}} \boldsymbol{X}_{j(K, i)}(t) \otimes \nabla \widehat{\varphi}_{i}(\widehat{\boldsymbol{x}}) .
$$

Note that with this definition $J$ has the usual form:

$$
J_{k l}=\frac{\partial x_{k}}{\partial \widehat{x}_{l}}, \quad k, l=1 \ldots d .
$$

At $t=0$, we have $\Phi_{K_{0}}:=\Phi_{K}(\cdot, 0): \widehat{\boldsymbol{x}} \rightarrow \boldsymbol{x}_{0}$ with Jacobian $J_{0}(\widehat{\boldsymbol{x}}):=J(\widehat{\boldsymbol{x}}, 0)$. Later in the text, we also use the mapping $\Phi_{K} \circ \Phi_{K_{0}}^{-1}: \boldsymbol{x}_{0} \rightarrow \boldsymbol{x}$ with Jacobian $J J_{0}^{-1}$. A realization of the mapping $\left(\Phi_{K}\right)_{K \in \mathcal{K}_{k}}$ using the $Q_{2}$ space is shown in Figure 2 For instance, looking at this figure, we understand that (33) tells us that we can obtain the position of the black points on the right side of the current element by using only the positions of the red dots on the right side of the reference cell.

\subsection{Length scales}

Artificial viscosity coefficients $(\lambda, \nu)$ must scale like a speed times a distance. This is usually done in Eulerian methods by defining (i) a mesh dependent length scale and (ii) a shock-capturing quantity, for example the entropy production. We are going to adapt this technique to the Lagrangian situation. The key difference is that now the mesh moves and the notion of meshsize is dynamic and therefore must be revised. In this subsection we define three different length 

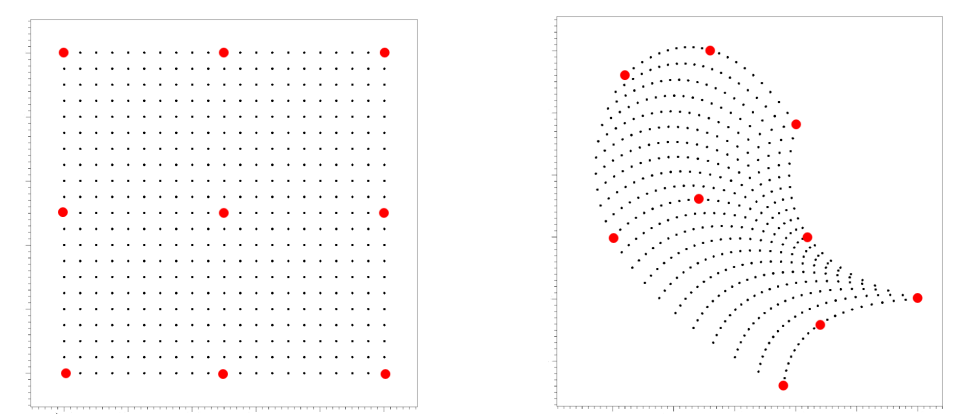

Figure 2: Example of a $Q_{2}$ mapping between the reference and the current element.

scales and we match them with specific coefficients in the next subsection. All the scales are defined pointwise in order to be computable at Gauss points and be compatible with the usage high order polynomial spaces.

We start by defining a smooth initial mesh length scale function $h_{0}(\boldsymbol{x})$ : (i) On each initial cell $K_{0}$, we define $h^{*}(\boldsymbol{x})=\frac{1}{k}\left|K_{0}\right|^{\frac{1}{d}}$ for all $\boldsymbol{x} \in K_{0}$ and set $h_{\text {min }}^{*}=\min _{\boldsymbol{x} \in \Omega_{0}} h^{*}(\boldsymbol{x})$, where recall that $k$ is the degree of the polynomials in $Q_{k}$. (ii) If $h^{*}(\boldsymbol{x})$ is smooth (i.e., the initial mesh is uniform), then we set $h_{0}=$ $h^{*}$, otherwise $h_{0}$ is computed by a smoothing procedure with some smoothing constant $\varepsilon$ :

$$
\int_{\Omega_{0}} h_{0}(\boldsymbol{x}) \varphi(\boldsymbol{x}) \mathrm{d} \boldsymbol{x}+\left(\varepsilon h_{\min }^{*}\right)^{2} \int_{\Omega_{0}} \nabla h_{0}(\boldsymbol{x}) \cdot \nabla \varphi(\boldsymbol{x}) \mathrm{d} \boldsymbol{x}=\int_{\Omega_{0}} h^{*}(\boldsymbol{x}) \varphi(\boldsymbol{x}) \mathrm{d} \boldsymbol{x} .
$$

We approximate $h_{0}$ in the same finite element space as the dependent variables.

Once $h_{0}(\boldsymbol{x})$ is known, we define three length scales by using the mapping $\Phi_{K} \circ \Phi_{K_{0}}^{-1}: \boldsymbol{x}_{0} \rightarrow \boldsymbol{x}$ with Jacobian $J J_{0}^{-1}:$

1. Recalling that $\|\cdot\|_{\ell^{2}}$ is the Euclidean norm in $\mathbb{R}^{d}, h_{1}(\boldsymbol{x})$ is defined as a perturbation of the initial mesh in the direction of the current motion $\boldsymbol{u}(\boldsymbol{x}, t)$ :

$$
h_{1}\left(\boldsymbol{x}\left(\boldsymbol{x}_{0}, t\right)\right)=h_{0}\left(\boldsymbol{x}_{0}\right) \frac{\left\|J J_{0}^{-1}\left(\boldsymbol{x}_{0}\right) \boldsymbol{u}(\boldsymbol{x}, t)\right\|_{\ell^{2}}}{\|\boldsymbol{u}(\boldsymbol{x}, t)\|_{\ell^{2}}} .
$$

2. $h_{2}(\boldsymbol{x})$ is defined as in Dobrev et al. [16, i.e., it is a perturbation of the initial mesh in the direction of maximal compression:

$$
h_{2}\left(\boldsymbol{x}\left(\boldsymbol{x}_{0}, t\right)\right)=h_{0}\left(\boldsymbol{x}_{0}\right) \frac{\left\|J J_{0}^{-1}\left(\boldsymbol{x}_{0}\right) \boldsymbol{s}(\boldsymbol{x})\right\|_{\ell^{2}}}{\|\boldsymbol{s}(\boldsymbol{x})\|_{\ell^{2}}} .
$$

where $s(\boldsymbol{x})$ is the eigenvector corresponding to the smallest eigenvalue $\mu(\boldsymbol{x})$ of $\nabla^{s} \boldsymbol{u}(\boldsymbol{x})$. Here $\mu(\boldsymbol{x})$ is a measure of the maximal compression (or minimal expansion if the value is positive), and $\boldsymbol{s}(\boldsymbol{x})$ is the direction in which this compression occurs. 
3. $h_{3}(\boldsymbol{x})$ is defined to be the measure of the volume change at $\boldsymbol{x}$ :

$$
h_{3}\left(\boldsymbol{x}\left(\boldsymbol{x}_{0}, t\right)\right)=h_{0}\left(\boldsymbol{x}_{0}\right)\left|\operatorname{det}\left(J J_{0}^{-1}\left(\boldsymbol{x}_{0}\right)\right)\right| .
$$

Note that $h_{1}$ and $h_{2}$ are direction-dependent, whereas $h_{3}$ is not. These functions are time-dependent and are not represented in the finite element space, their values are computed whenever needed (usually at quadrature points).

\subsection{Viscosity coefficients}

As discussed in the previous subsection, the artificial viscosity coefficients $(\lambda, \nu)$ must be an appropriate combination of a length scale and a shock detector, and they should be computed at each quadrature point. We first define the "first-order" viscous coefficients. Let $\boldsymbol{x}^{n}$ be the position of the quadrature point of interest at time $t_{n}$, and let $c_{\text {visc }} \geq 0$ be a tunable constant. We propose two options corresponding to $h_{1}$ and $h_{2}$ :

\section{- Option 1:}

$$
\begin{aligned}
& \lambda_{1}^{\mathrm{visc}}:=c_{\mathrm{visc}} h_{1}\left(\boldsymbol{x}^{n}\right)\left\|\boldsymbol{u}^{n}\left(\boldsymbol{x}^{n}\right)\right\|_{\ell^{2}}, \\
& \nu_{1}^{\mathrm{visc}}:=c_{\mathrm{visc}} h_{1}\left(\boldsymbol{x}^{n}\right)\left(a\left(\boldsymbol{x}^{n}\right)+\left\|\boldsymbol{u}^{n}\left(\boldsymbol{x}^{n}\right)\right\|_{\ell^{2}}\right),
\end{aligned}
$$

where $a\left(\boldsymbol{x}^{n}\right)$ is the sound speed. These quantities are not shock detectors since diffusion is active everywhere the velocity is nonzero.

- Option 2: (which is used in Dobrev et al. [16]):

$$
\begin{aligned}
\lambda_{2}^{\text {visc }}: & =c_{\text {visc }} h_{2}^{2}\left(\boldsymbol{x}^{n}\right)\left|\mu\left(\boldsymbol{x}^{n}\right)\right|, \\
\nu_{2}^{\text {visc }}: & = \begin{cases}c_{\mathrm{visc}} h_{2}\left(\boldsymbol{x}^{n}\right)\left(a\left(\boldsymbol{x}^{n}\right)+h_{2}\left(\boldsymbol{x}^{n}\right)\left|\mu\left(\boldsymbol{x}^{n}\right)\right|\right) & \mu\left(\boldsymbol{x}^{n}\right)<0, \\
c_{\mathrm{visc}} h_{2}^{2}\left(\boldsymbol{x}^{n}\right)\left|\mu\left(\boldsymbol{x}^{n}\right)\right| & \text { otherwise } .\end{cases}
\end{aligned}
$$

where recall that $\mu(\boldsymbol{x}) \leq 0$ in compressive regions. Note that the above definition is formally less diffusive than the first one, but it is nevertheless first-order in compressive regions. Note also that both $\lambda_{2}^{\text {visc }}$ and $\nu_{2}^{\text {visc }}$ are small when the velocity is close to a constant, which is the case, for instance, in contact regions.

Then we define "non-linear" viscosity coefficients by using the property that the entropy production is zero in regions where the solution is smooth (at the continuous level) and non-zero in singular regions. In the Lagrangian frame of reference, equation (11) becomes

$$
\frac{d S}{d t}+S \nabla \cdot \boldsymbol{u}=0
$$

where $S$ is defined in 12 . Then at the discrete level, we define

$$
D:=\frac{S^{n}-S^{n-1}}{t_{n}-t_{n-1}}+S^{n} \nabla \cdot \boldsymbol{u}^{n}\left(\boldsymbol{x}^{n}\right),
$$

where $S^{n}=S\left(\rho^{n}, e^{n}\right)$ is the entropy functional at $t_{n}$. If there is an energy source as in (17) then 42 becomes

$$
D:=\frac{S^{n}-S^{n-1}}{t_{n}-t_{n-1}}+S^{n} \nabla \cdot \boldsymbol{u}^{n}\left(\boldsymbol{x}^{n}\right)+\frac{Q}{f^{\prime}\left(s^{n}\right)} e^{n},
$$


where $s^{n}=s\left(\rho^{n}, e^{n}\right)$ is the specific entropy at $t_{n}$ and $Q$ is the source contribution at the quadrature point of interest. The time derivative of $S$ can be approximated by higher-order backward differencing; this is what is actually done in the convergence tests reported below for smooth solutions. The highorder viscosity coefficient is defined to be

$$
\nu^{\mathrm{entr}}:=\frac{c_{\mathrm{entr}} h_{3}^{2}\left(\boldsymbol{x}^{n}\right)|D|}{\left\|S^{n}-\overline{S^{n}}\right\|_{L \infty\left(\Omega\left(t_{n}\right)\right)}+\epsilon \overline{S^{n}}}, \quad \overline{S^{n}}:=\frac{1}{\left|\Omega\left(t_{n}\right)\right|} \int_{\Omega\left(t_{n}\right)} S^{n} d x,
$$

where $c_{\text {entr }}$ is a tunable constant and $\epsilon=10^{-10}$. Finally the so-called entropy viscosity is obtained by taking the minimum of the first-order and high-order viscosities. We consider two options:

- Option 1:

$$
\lambda_{1}^{n}:=\min \left(\lambda_{1}^{\mathrm{visc}}, \nu^{\mathrm{entr}}\right), \quad \nu_{1}^{n}:=\min \left(\nu_{1}^{\mathrm{visc}}, \nu^{\mathrm{entr}}\right) .
$$

- Option 2:

$$
\lambda_{2}^{n}:=\min \left(\lambda_{2}^{\text {visc }}, \nu^{\text {entr }}\right), \quad \nu_{2}^{n}:=\min \left(\nu_{2}^{\text {visc }}, \nu^{\text {entr }}\right) .
$$

Note that the definitions (45) and (46) are dimensionally coherent since the quantities $\lambda_{1}^{\text {visc }}, \lambda_{2}^{\text {visc }}, \nu_{1}^{\text {visc }}, \nu_{2}^{\text {visc }}$, and $\nu^{\text {entr }}$ scale like a speed times a length. The heuristics for the above definitions is that we expect the first-order viscosity to be the active in shocks and contacts and the high-order vanishing viscosity to be active in the smooth regions.

Using appropriate combinations of length scales and shock detectors is essential for avoiding incorrect mesh behavior, see definitions (40), (41), (44). The first-order shock detectors depend on the relative orientation of the velocity with respect to the mesh, whereas the entropy production coefficient and its associated length scale are direction-independent. In Option 1, the length scales in (44) are $h_{1}$ and $h_{3}$, but they are $h_{2}$ and $h_{3}$ in Option 2. And example of incorrect combination of length scales is shown in Figure 3 The left panel shows the mesh obtained by solving the Sedov problem (see 4.3 for details) using Option 2 with the correct combination of length scales (i.e., $h_{2}$ in (41)). The right panel shows the result obtained by using $h_{1}$ instead on $h_{2}$ in (41).

\subsection{Consistency with general viscosity requirements}

Here we comment on the compatibility of the viscosity tensors with the general requirements for artificial tensor viscosities stated in Shashkov and Campbell [42] and Kolev and Rieben [26. We use the terminology from Kolev and Rieben [26]:

- The viscous terms must be invariant under orthogonal transformations of $\boldsymbol{x}$ and $\boldsymbol{u}$ : the method satisfies this requirement at the discrete level; the details are given in 6.1 . 

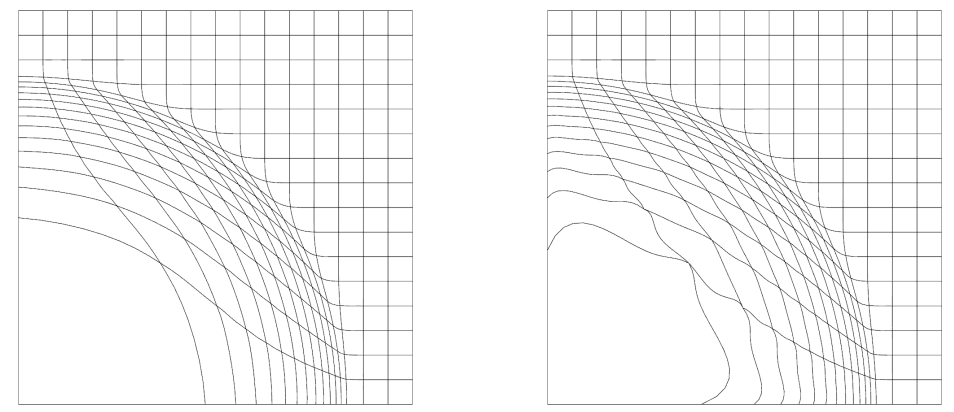

Figure 3: Resulting meshes from applying the Option 2 coefficients to a $Q_{4}$ position function with $h_{3}$ (left panel) and $h_{1}$ (right panel) in equation 44.

- For the specific entropy s, see (9), we must have $\frac{d s}{d t} \geq 0$ : the regularization satisfies a weaker version of this requirement, namely the minimum principle on the specific entropy (16) on continuous level. This result is established in Guermond and Popov [20] (Theorem 3.5).

- The regularized system must be Galilean invariant: the system in Lagrangian form (24)-(26) is Galilean invariant. All the viscosity coefficients in option 2 , the entropy production $D, h_{2}, h_{3}$ satisfy the requirement at the discrete level. Option 1 and $h_{1}$ are not Galilean invariant, though, since they depend explicitly on $\boldsymbol{u}$.

- The artificial viscosity must preserve radial symmetry: the symmetry preservation of our method is presented by the numerical tests in Sections 4.3 , 4.4, 4.5. In general, achieving radial symmetry on fully-discrete level is a difficult task, because that would require the artificial viscosity, and in particular its length scale part, to be radially symmetric. Length scales, however, typically depend on initial cell sizes and directions, which are not in general radially symmetric. Exact radial symmetry can be achieved in special cases, for example by constructing the initial mesh with curved radial cells obtained from each other by orthogonal transformation, see Section 8.2 in Kolev and Rieben [26].

- The artificial dissipation in the momentum equation must be zero for linear velocity (uniform contractions, rigid rotations) and all the artificial viscosity terms must be zero in regions of expansion: these requirements are not satisfied exactly but are satisfied up to the consistency error of the method. More precisely the entropy viscosity (44) is $\mathcal{O}\left((\Delta t+h) h^{2}\right)$ in smooth regions by construction. (It can be made $\mathcal{O}\left(\Delta t^{2}+h\right) h^{2}$ by using Crank-Nicolson's or BDF2 approximation in 43 , see 4.1 )

\subsection{Time discretization}

We discretize the time derivatives of $(\rho, \boldsymbol{u}, e, \boldsymbol{x})$ by using a standard explicit Runge-Kutta method (i.e., either RK3 or RK4). To be generic, we assume the 
method is composed of $r$ stages and be fully described by its Butcher tableau 1. Let $\boldsymbol{v}=(\rho, \boldsymbol{u}, e, \boldsymbol{x})$ be the discrete solution at time $t$. We introduce the

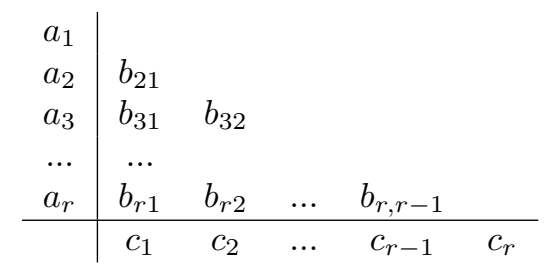

Table 1: Butcher tableau for a $r$-stage explicit RK method.

discrete operators $W_{\rho}, \boldsymbol{W}_{\boldsymbol{u}}, W_{e}, \boldsymbol{W}_{\boldsymbol{x}}$ defined by solving the following discrete weak versions of (28)-(30):

$$
\begin{aligned}
\int_{\Omega(t)} W_{\rho}(\boldsymbol{v}, t) \varphi \mathrm{d} \boldsymbol{x} & =\int_{\Omega(t)}(-\rho \nabla \cdot \boldsymbol{u} \varphi-(\lambda \nabla \rho) \cdot \nabla \varphi) \mathrm{d} \boldsymbol{x}, \\
\int_{\Omega(t)} \rho\left[W_{\boldsymbol{u}}(\boldsymbol{v}, t)\right]_{m} \varphi \mathrm{d} \boldsymbol{x} & =\int_{\Omega(t)}\left(\lambda \nabla u_{m} \cdot \nabla \rho \varphi-\nu \rho \nabla u_{m} \cdot \nabla \varphi-\frac{\partial p}{\partial x_{j}} \varphi\right) \mathrm{d} \boldsymbol{x}, \quad m=1 \ldots d, \\
\int_{\Omega(t)} \rho W_{e}(\boldsymbol{v}, t) \varphi \mathrm{d} \boldsymbol{x} & =\int_{\Omega(t)}\left(\nu \rho\|\nabla \boldsymbol{u}\|_{\ell^{2}}^{2} \varphi-\lambda \rho \nabla e \cdot \nabla \varphi+(\lambda \nabla e \cdot \nabla \rho) \varphi-p \nabla \cdot \boldsymbol{u} \varphi\right) \mathrm{d} \boldsymbol{x}, \\
\boldsymbol{W}_{\boldsymbol{x}}(\boldsymbol{v}, t) & =\boldsymbol{u} .
\end{aligned}
$$

Then upon denoting $\boldsymbol{W}(\boldsymbol{v}, t):=\left(W_{\rho}, \boldsymbol{W}_{\boldsymbol{u}}, W_{e}, \boldsymbol{W}_{\boldsymbol{x}}\right)$, the solution $\boldsymbol{v}$ is updated at time $t_{n+1}$ by

$$
\boldsymbol{v}^{n+1}=\boldsymbol{v}^{n}+\Delta t \sum_{i=1}^{r} c_{i} \boldsymbol{k}_{i}, \quad \boldsymbol{k}_{i}:=\boldsymbol{W}\left(\boldsymbol{v}^{n}+\Delta t \sum_{j=1}^{r} b_{i j} \boldsymbol{k}_{j}, t_{n}+a_{i} \Delta t\right) .
$$

\subsection{Time step control}

Because of the finite speed of propagation in hyperbolic problems, the time step for explicit methods is restricted by a CFL condition. We then introduce the CFL number $c$ and control the time step by using the following heuristic technique:

1. Given $\left(\rho^{0}, \boldsymbol{u}^{0}, e^{0}, \boldsymbol{x}^{0}\right)$ at time $t_{0}$, compute the first time step:

$$
\Delta t=\min _{\boldsymbol{x} \in \Omega_{n}} \frac{c_{\mathrm{cff}} h_{2}(\boldsymbol{x})}{\sqrt{\gamma T^{0}(\boldsymbol{x})}+\left|\boldsymbol{u}^{0}(\boldsymbol{x})\right|} .
$$

2. Use $\Delta t$ to compute the update $\left(\rho^{n+1}, \boldsymbol{u}^{n+1}, e^{n+1}, \boldsymbol{x}^{n+1}\right)$ and compute:

$$
\Delta t^{*}=\min _{\boldsymbol{x} \in \Omega_{n+1}} \frac{c_{\mathrm{cff}} h_{2}(\boldsymbol{x})}{\sqrt{\gamma T^{n+1}(\boldsymbol{x})}+\left|\boldsymbol{u}^{n+1}(\boldsymbol{x})\right|} .
$$


3. If $\Delta t^{*}<\Delta t \Rightarrow \Delta t=0.9 \Delta t$, discard the update and go to step 2 (i.e., repeat the step).

4. Else, set $n=n+1$. If $\Delta t^{*}>1.25 \Delta t$ then set $\Delta t=1.02 \Delta t$. Set $t=t+\Delta t$ and go to step 2 .

\section{Numerical tests}

In this section we illustrate the behavior of the method on standard Lagrangian hydrodynamics test cases with known exact solutions. First we use a smooth solution to estimate the convergence properties of the method. Then, we use a one-dimensional Riemann problem to demonstrate the shock-capturing properties of the entropy production-based viscosity coefficients and the nonoscillating behavior of the method in contact regions. Finally, we turn to twodimensional shock problems to test the symmetry properties of the method.

For all the test cases, we solve the linear systems associated with the mass matrix by using a conjugate gradient algorithm with a diagonal Jacobi preconditioner. The list of the parameters used in each of the test cases is reported in $\$ 6.2$.

The method is made parallel by using the parallel finite element method library MFEM [36]. The results are visualized by using the OpenGL visualization tool GLVis [18.

\subsection{D Taylor-Green vortex}

The goal of this test case is to demonstrate that the method achieves highorder convergence rates on smooth solutions. This is essentially a consistency test. All the simulations are done by keeping the viscosity terms active in equations (28)-(30) (i.e., we do not set them to zero explicitly). This confirms the convergence to zero of the entropy production-based viscosity coefficients on discrete level.

The 2D Taylor-Green vortex test case is designed so that the solution in Eulerian coordinates is smooth and time-independent. This is done by introducing a manufactured source of internal energy $Q$

$$
\rho \frac{d e}{d t}=-p \nabla \cdot \boldsymbol{u}+Q
$$

Using the following initial conditions and source

$$
\begin{aligned}
\rho_{0}\left(\boldsymbol{x}_{0}\right) & =1, \quad \boldsymbol{u}_{0}\left(\boldsymbol{x}_{0}\right)=\left(\sin \left(\pi x_{0}\right) \cos \left(\pi y_{0}\right)-\cos \left(\pi x_{0}\right) \sin \left(\pi y_{0}\right)\right), \\
p_{0}\left(\boldsymbol{x}_{0}\right) & =\frac{\rho}{4}\left(\cos \left(2 \pi x_{0}\right)+\cos \left(2 \pi y_{0}\right)+1, \quad \gamma=\frac{5}{3},\right. \\
Q & =\frac{3 \pi}{8}(\cos (3 \pi x) \cos (\pi y)-\cos (\pi x) \cos (3 \pi y)),
\end{aligned}
$$

the exact solution is $\rho(\boldsymbol{x}, t)=\rho_{0}(\boldsymbol{x}), \boldsymbol{u}(\boldsymbol{x}, t)=\boldsymbol{u}_{0}(\boldsymbol{x}), e(\boldsymbol{x}, t)=e_{0}(\boldsymbol{x})$ for all $\boldsymbol{x} \in \Omega(t)$. 
For smooth solutions, the order of accuracy of the method depends on the polynomial degree of the approximation space and on how fast the artificial viscosity terms converges to zero. In order to minimize the consistency error in the entropy residual equation (43), we approximate the time derivative of the entropy by using BDF2. This, together with the $h_{3}^{2}$ scaling in (44) produces a consistency error of 4th order, i.e., the artificial viscosity is formally fourth order. One can use backward differencing with more points in 43 in order to achieve orders higher than 4.

We run the problem up to $t=0.5$ with the boundary condition $\boldsymbol{u} \cdot \boldsymbol{n}=0$. The initial and final meshes and the corresponding velocity magnitudes with a $Q_{4}$ finite element space are shown in Figure 4. We compare a $Q_{1}$ and a $Q_{4}$
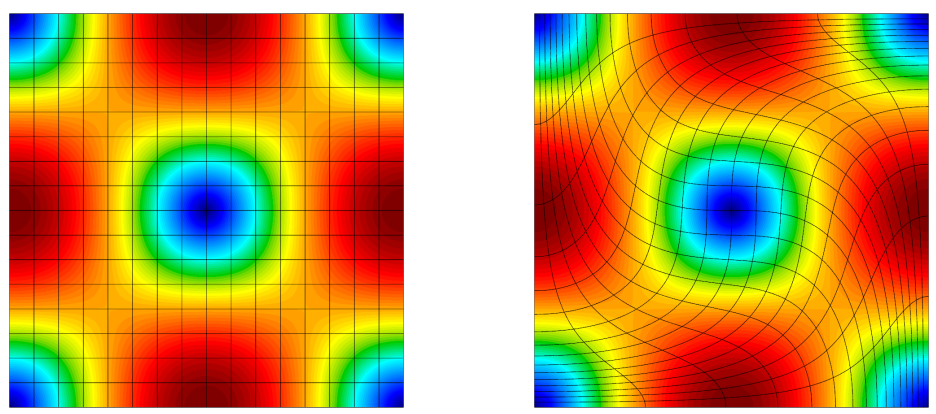

Figure 4: Velocity magnitude on the initial mesh (left panel), and on the final mesh (right panel) computed with the $Q_{4}$ FE space using $16 \times 16$ cells for the 2 D Taylor-Green vortex problem.
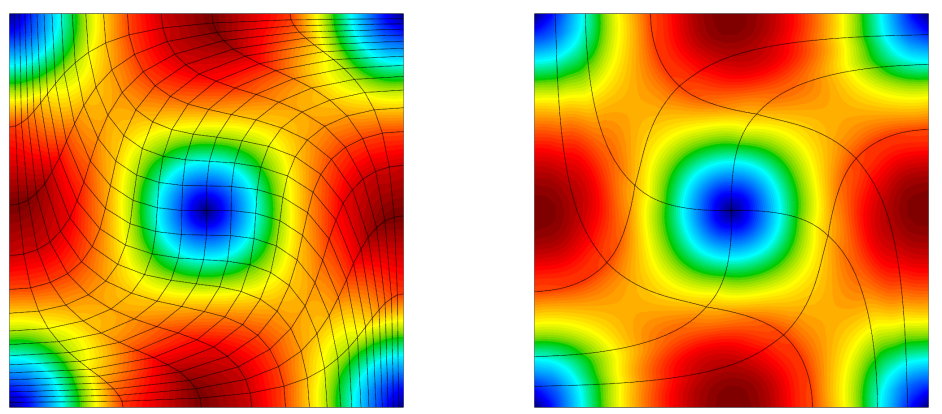

Figure 5: Final mesh and velocity magnitude for a $Q_{1}$ simulation with $16 \times 16$ cells (left panel), and for a $Q_{4}$ simulation with $4 \times 4$ cells (right panel) for the 2 D Taylor-Green vortex problem.

simulation with similar number of degrees of freedom in Figure 5. Although it may not be clearly visible on the figure, the convergence rates reported in Table 2 show that the resolution of the $Q_{4}$ space is far superior to that of the $Q_{1}$ space. In Table 2 we show $L^{1}$-convergence rates for the velocity computed with finite element spaces of different order. The expected high-order convergence rates are observed. Note that the $Q_{4}$ approximation is limited to fourth order since we used BDF2 to evaluate the time derivative of the entropy in the entropy residual. 


\begin{tabular}{|ccccccccc|}
\hline & $Q_{1}$ & \multicolumn{3}{c|}{$Q_{2}$} & \multicolumn{2}{c|}{$Q_{3}$} & & $Q_{4}$ \\
\hline$h_{0}$ & $L^{1}$ error & rate & $L^{1}$ error & rate & $L^{1}$ error & rate & $L^{1}$ error & rate \\
\hline $1 / 4$ & $4.08 \mathrm{e}-1$ & & $3.61 \mathrm{e}-2$ & & $1.87 \mathrm{e}-2$ & & $6.22 \mathrm{e}-3$ & \\
$1 / 8$ & $7.39 \mathrm{e}-2$ & 2.46 & $6.13 \mathrm{e}-3$ & 2.55 & $2.53 \mathrm{e}-3$ & 2.88 & $2.35 \mathrm{e}-4$ & 4.72 \\
$1 / 16$ & $1.07 \mathrm{e}-2$ & 2.78 & $1.54 \mathrm{e}-3$ & 1.99 & $1.71 \mathrm{e}-4$ & 3.88 & $8.47 \mathrm{e}-6$ & 4.79 \\
$1 / 32$ & $1.96 \mathrm{e}-3$ & 2.44 & $3.99 \mathrm{e}-4$ & 1.94 & $6.69 \mathrm{e}-6$ & 4.67 & $7.14 \mathrm{e}-7$ & 3.56 \\
$1 / 64$ & $4.26 \mathrm{e}-4$ & 2.20 & $1.00 \mathrm{e}-4$ & 1.99 & $4.65 \mathrm{e}-7$ & 3.84 & $4.89 \mathrm{e}-8$ & 3.86 \\
$1 / 128$ & $1.00 \mathrm{e}-4$ & 2.09 & $2.42 \mathrm{e}-5$ & 2.04 & $4.89 \mathrm{e}-8$ & 3.24 & $3.23 \mathrm{e}-9$ & 3.92 \\
\hline
\end{tabular}

Table 2: $L^{1}$-norm of the error on the velocity and convergence rates for the $2 \mathrm{D}$ Taylor-Green vortex problem, $c_{\mathrm{cfl}}=0.1$.

\section{2. $1 D$ Sod tube}

The goal of this test is to demonstrate the capabilities of the method to capture shocks and contact discontinuities properly. This is a 1D Riemann problem developing a rarefaction, a contact and a shock wave. The initial computational domain is $[0,1]$. The initial conditions are:

$\rho\left(x_{0}\right)=\left\{\begin{array}{ll}1.0 & x_{0} \leq 0.5, \\ 0.125 & \text { otherwise, }\end{array}, \quad \boldsymbol{u}\left(x_{0}\right)=0, p\left(x_{0}\right)=\left\{\begin{array}{ll}1.0 & x_{0} \leq 0.5, \\ 0.1 & \text { otherwise, }\end{array}, \gamma=1.4\right.\right.$

The final time is 0.2 and we enforce $\boldsymbol{u} \cdot \boldsymbol{n}=0$ at the boundary.

We first discuss the effects of using the viscosity coefficients defined in Option 2, see 41. In Figure 6 we show density field (on the left panel) and the magnitude of the compression measure $\mu(\boldsymbol{x})$ at the final time, see (41). The simulation is done with $\mathbb{Q}_{1} \mathrm{FE}$ on a mesh composed of 128 cells. We observe that Option 2 provides sufficient dissipation in the shock region, but almost none in the contact. The reason for this is that the compression measure $\mu(\boldsymbol{x})$ is based only on the gradient of the velocity, and the velocity is almost constant in the region of the contact. The oscillations in density, pressure and energy around the contact cause some small velocity gradients, but the generated compression is too small to dampen those oscillations. Next we present in Figure 7 results
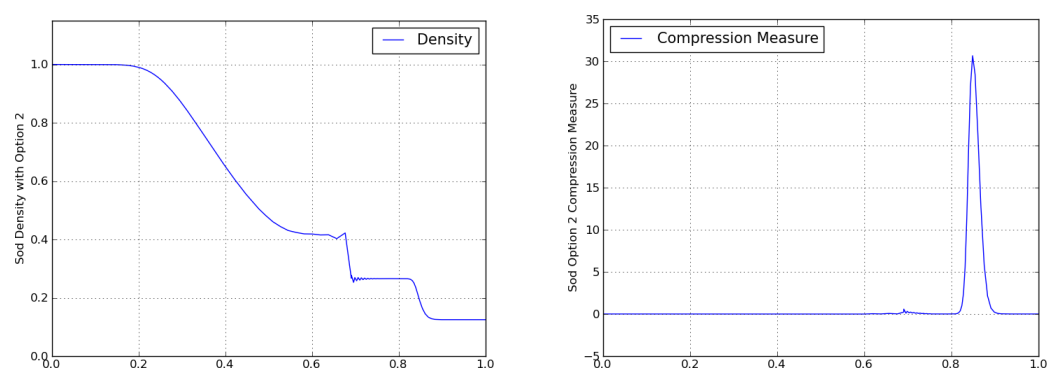

Figure 6: 1D Sod tube problem using Option 2 for the viscosity coefficients. Density field (left panel) and compression measure (right panel) 
obtained by using Option 1 for the viscosity. On the left panel of the Figure we show the density field computed with $Q_{1}$ and $Q_{4} \mathrm{FE}$ spaces using the firstorder viscosity only, i.e., $c_{\text {entr }}=\infty$ in 40 . There are 256 and 1024 cells for the $Q_{4}$ and $Q_{1}$ spaces, respectively, so that the numbers of degrees of freedom are almost identical for the two spaces. We observe that for both the $Q_{1}$ and $Q_{4}$ approximations, the definition 40 provides enough diffusion throughout the domain. In the center and in the right panel of the Figure 7 we show the density and pressure fields obtained with the entropy viscosity, see 45). The results are sharper than those generated by the first-order viscosity and there are no oscillations in the contact region, meaning that the entropy production provides sufficient dissipation. On the left figure, where we use the first-order viscosity, we observe that the results for the $Q_{1}$ and $Q_{4} \mathrm{FE}$ spaces are essentially the same.
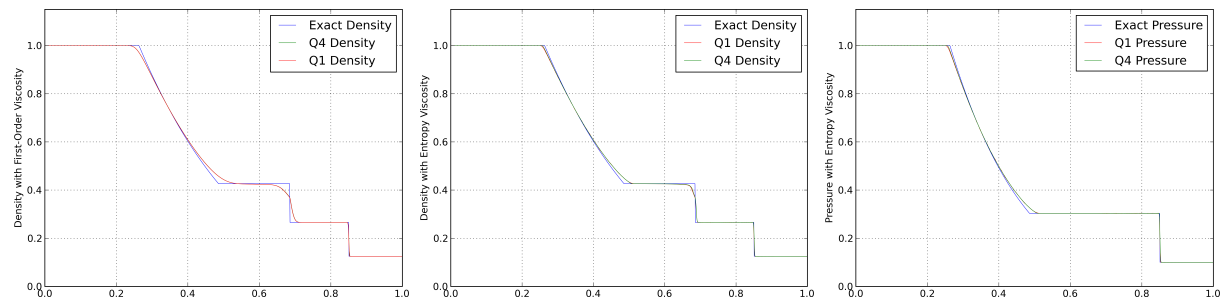

Figure 7: Sod shocktube, $Q_{1}$ vs. $Q_{4}$ approximation, both with Option 1. Density field computed with the first-order viscosity (left panel). Density (center panel) and pressure (right panel) computed with the entropy viscosity.

In Figure 8 we compare the $L^{2}$-projections of the first order viscosity field (40), which was used to generate the results in the left panel of Figure 7, and the entropy-based viscosity field (44) which was used to generate the results in the center and right panels of Figure 7. We observe that in the smooth regions the entropy production coefficient is the active part of the minimum in (45), and the first order coefficient is the active part in the shock, thereby confirming the heuristic argument that lead to the notion of entropy-based viscosity.

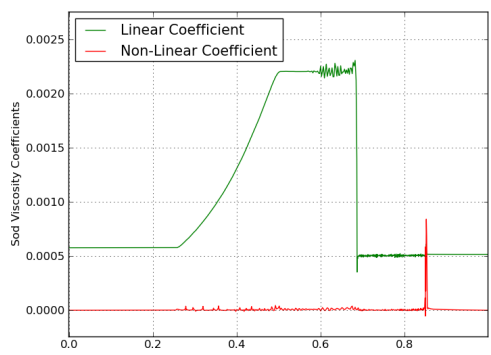

Figure 8: 1D Sod tube problem. Comparison between the $L^{2}$-projections of the first-order viscosity and the high-order viscosity in Option 1.

We show In table 3 the convergence rates in the $L^{1}$-norm for the density computed with $Q_{1}, Q_{2}$ and $Q_{4}$ spaces. The rows are aligned so that the rates 
in each row are computed with approximately the same number of degrees of freedom. We observe that the errors and rates for all the finite element spaces are similar, they are close to the optimal rate of 1 .

\begin{tabular}{|ccccccccc|}
\hline & $Q_{1}$ & \multicolumn{3}{c|}{$Q_{2}$} & & \multicolumn{3}{c|}{$Q_{4}$} \\
\hline$h_{0}$ & $L^{1}$ error & rate & $h_{0}$ & $L^{1}$ error & rate & $h_{0}$ & $L^{1}$ error & rate \\
\hline $1 / 64$ & 0.02194 & & $1 / 32$ & 0.02216 & & $1 / 16$ & 0.02190 & \\
$1 / 128$ & 0.01319 & 0.73 & $1 / 64$ & 0.01335 & 0.73 & $1 / 32$ & 0.01264 & 0.79 \\
$1 / 256$ & 0.00723 & 0.86 & $1 / 128$ & 0.00747 & 0.83 & $1 / 64$ & 0.00701 & 0.85 \\
$1 / 512$ & 0.00382 & 0.92 & $1 / 256$ & 0.00394 & 0.92 & $1 / 128$ & 0.00383 & 0.87 \\
$1 / 1024$ & 0.00199 & 0.94 & $1 / 512$ & 0.00204 & 0.95 & $1 / 256$ & 0.00207 & 0.88 \\
\hline
\end{tabular}

Table 3: $L^{1}$-norm of error on density and convergence rates for the $1 \mathrm{D}$ Sod tube problem.

\subsection{D Sedov explosion}

The Sedov explosion, introduced in Sedov [40, is a standard problem to test the ability of codes to preserve the radial symmetry of shocks. The initial conditions are

$$
\rho\left(\boldsymbol{x}_{0}\right)=1, \quad \boldsymbol{u}\left(\boldsymbol{x}_{0}\right)=0, \quad e\left(\boldsymbol{x}_{0}\right)=\delta_{0}, \int_{\Omega_{0}} e\left(\boldsymbol{x}_{0}\right) \mathrm{d} \boldsymbol{x}_{0}=E_{\text {tot }}, \quad \gamma=1.4 .
$$

where $\delta_{0}$ is the Dirac measure supported at $\boldsymbol{x}_{0}=0$, and $E_{\text {tot }}=1$ if the origin $\boldsymbol{x}_{0}=0$ is in the interior of $\Omega_{0}$. The internal energy deposited at the origin converts into kinetic energy thereby creating an expanding shock wave. The problem is usually run up to $t=1.0$.

First we discuss results obtained by using $Q_{2} \mathrm{FE}$ spaces on a Cartesian uniform mesh for the sector $\Omega_{0}=[0,1.2] \times[0,1.2]$ with $64 \times 64$ cells. Note that since one quarter of the Dirac measure is supported in $\Omega_{0}$, the initial energy is $E_{\mathrm{tot}}=\frac{1}{4}$ for this case. We enforce the symmetry condition $\boldsymbol{u} \cdot \boldsymbol{n}=0$ on the left and bottom boundaries. In the left panel of Figure 9 we compare the exact density and the approximate density fields obtained by using the Option 1, see (45), and Option 2, see (46), for the viscosity coefficients. This figure shows the nodal value of the density as a function of the radial distance to the origin for all the nodes in the mesh. This representation is meant to evaluate the radial symmetry of the approximate solutions. There is no clear difference between the two options. The results shown in the right panel compare the density obtained with the first order viscosity (41) and the entropy viscosity from Option 2, (46). We see that the entropy-based viscosity 46 is sharper than the first-order one (the maximum is higher).

On Figure 10 we show the $L^{2}$-projection of the entropy residual, i.e., $|D|$ in (44). Note that the residual $|D|$ focuses very well in the shock as expected.

On Figure 11 we show the mesh, the density, the velocity magnitude and pressure obtained by the Option 2 viscosity (46). The shock is properly captured and the motion of the mesh is correct. 

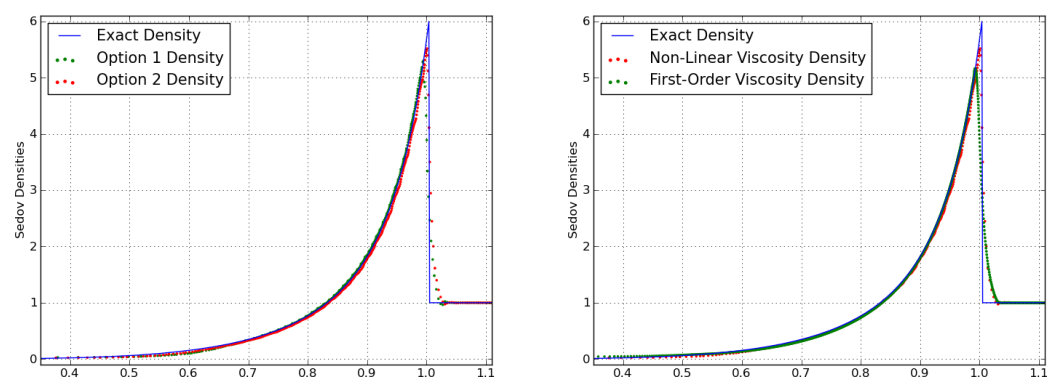

Figure 9: 2D Sedov problem at $t=1$. Density vs. radius for entropy viscosity from Option 1 and Option 2 (left panel). Density vs. for radius for entropy viscosity and first-order viscosity from Option 2 (right panel).
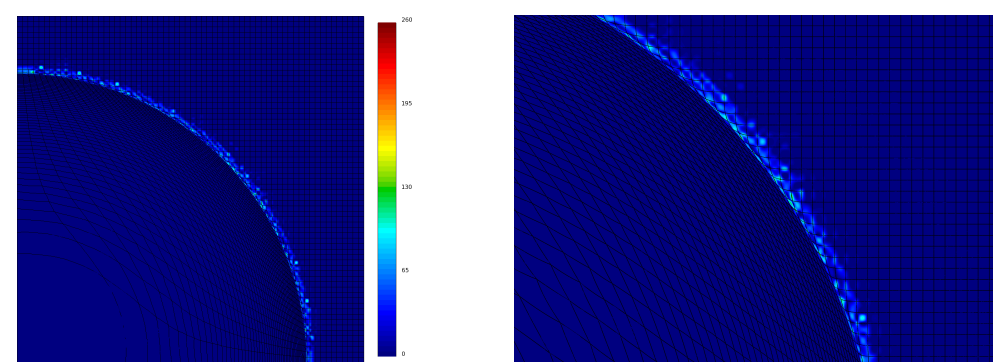

Figure 10: 2D Sedov problem at $t=1$. Mesh and $L^{2}$-projection of the entropy residual $|D|$ (left panel). Zoom at the shock region (right panel).
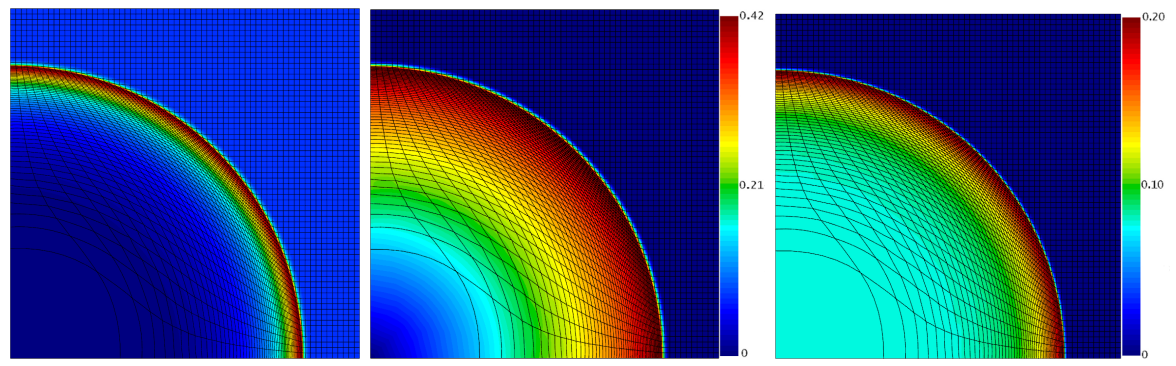

Figure 11: 2D Sedov explosion problem. Entropy viscosity solution at $t=1$ using Option 2: density (left), velocity magnitude (center), and pressure (right).

Next we consider a Cartesian non-uniform mesh for $\Omega_{0}=[-1.2,1.2] \times[-1.2,1.2]$. In this case the initial energy is $E_{\mathrm{tot}}=1.0$ since the Dirac measure at the origin is entirely supported in $\Omega_{0}$. We use $32 \times 64$ cells in quadrant \#1, $64 \times 64$ cells in quadrant \#2, $64 \times 32$ cells in quadrant $\# 3$, and $32 \times 32$ cells in quadrant \#4. The purpose of this test is to evaluate the ability of the scheme to preserve the radial symmetry of the solution in the presence of non uniformities in the mesh. The computation is done up to $t=1$ with $Q_{2}$ finite elements. We show in the left panel of Figure 12 the density and the mesh at $t=1$. We show in the right 
panel of the figure the distribution of the density as a function of the distance to the origin along the lines $|x|=|y|$ for each quadrant. The method performs satisfactorily since the radial symmetry is well preserved and the mesh motion is correct.
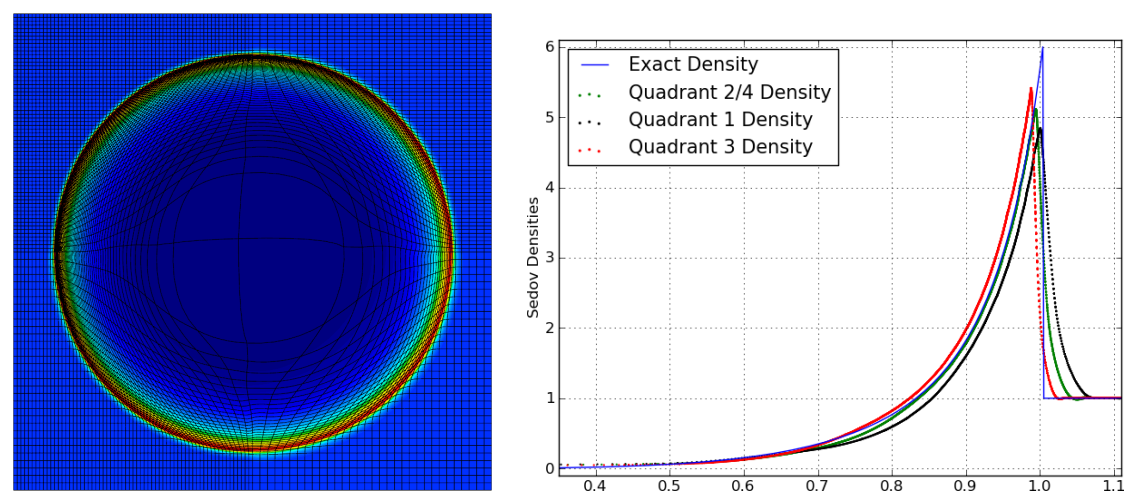

Figure 12: 2D Sedov problem on a non-uniform mesh. Density and mesh at $t=1$ (left panel), and density vs. radius along the lines $|x|=|y|$ (right panel).

\subsection{D Noh implosion}

The Noh implosion, introduced in Noh [37, is another benchmark problem tailored for testing the ability of numerical schemes to preserve symmetry. The initial conditions are

$$
\rho\left(\boldsymbol{x}_{0}\right)=1, \quad \boldsymbol{u}\left(\boldsymbol{x}_{0}\right)=-\frac{\boldsymbol{x}}{\|\boldsymbol{x}\|_{\ell^{2}}}, e\left(\boldsymbol{x}_{0}\right)=0, \quad \gamma=\frac{5}{3} .
$$

The initial velocity generates an outward traveling shock wave. This test is usually run up to $t=0.6$.

First we discuss results obtained by using $Q_{2}$ FE spaces on a uniform Cartesian mesh for the quadrant $\Omega_{0}=[0,1.2] \times[0,1.2]$. We enforce $\boldsymbol{u} \cdot \boldsymbol{n}=0$ on the left and bottom boundaries of $\Omega_{0}$. All the simulations reported hereafter are done with the Option 1 of the viscosity 40 . When using the same parameters for both options, Option 2 tangles the mesh and the simulation fails. It is possible to stabilize the computation with Option 2 by increasing $c_{\text {entr }}$, but in all the cases the results obtained with Option 1 are always less oscillatory and therefore the results from Option 2 are not reported. Figure 13 shows the mesh, the density (left), the velocity magnitude (center) and the pressure (right) obtained at $t=1$ by using the entropy viscosity from Option 1 (45) on a mesh composed of $64 \times 64$ cells. The left panel of Figure 14 shows the exact density and the densities computed on meshes composed of $32 \times 32,64 \times 64$ and $128 \times 128$ cells. The method is slightly oscillatory but convergent. In the two other panels are shown the $L^{2}$-projections of the high-order viscosity (center panel) and the first-order coefficient (right panel). Again we observe that the high-order viscosity is almost zero away from the shock region and it dominates the first 


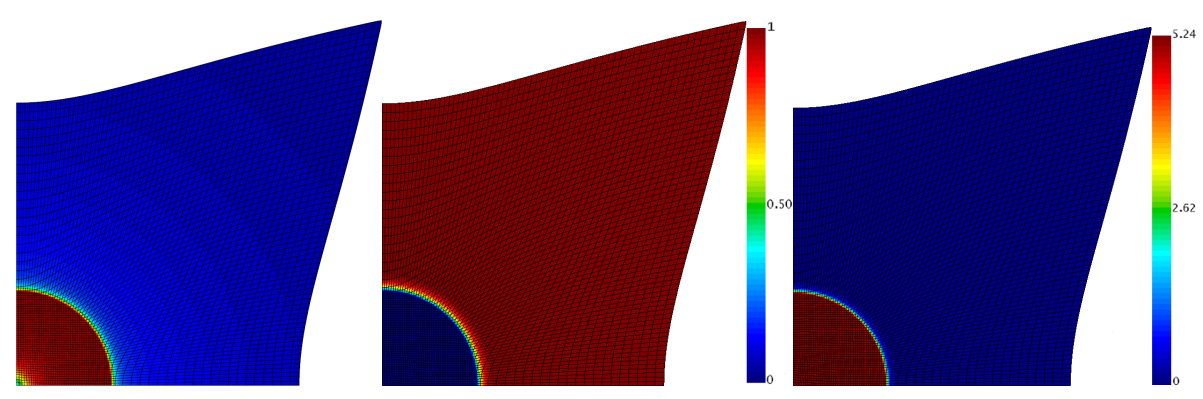

Figure 13: 2D Noh problem at $t=1$. Density (left panel), velocity magnitude (center panel), pressure (right panel) and $Q_{2}$ mesh with $64 \times 64$ cells.

order viscosity in the shock. This justifies the heuristic definition of the entropy viscosity in 45) and 46.

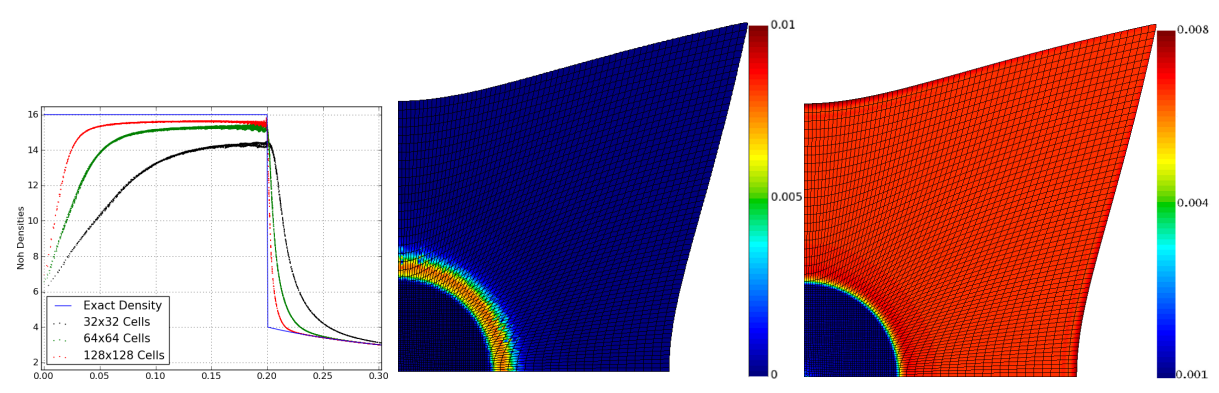

Figure 14: $2 \mathrm{D}$ Noh problem at $t=1$. Density vs. radius on different $Q_{2}$ meshes, $32 \times 32$, $64 \times 64$ and $128 \times 128$ (left panel). $L^{2}$-projections of the high-order viscosity (center panel) and first-order viscosity (right panel) from Option 1, 64×64 cells.

Next we consider a non-uniform Cartesian mesh with $\Omega_{0}=[-1.2,1.2] \times[-1.2,1.2]$. We use the $Q_{2}$ approximation and put $32 \times 64$ cells in quadrant $\# 1,64 \times 64$ cells in quadrant \#2, $64 \times 32$ cells in quadrant \#3, and $32 \times 32$ cells in quadrant \#4. The purpose of this mesh is to test again the ability of the scheme to preserve radial symmetry for radial shocks. The left panel of Figure 15 shows the density and the mesh at $t=1$. The right panel of the figure shows the density as a function of the distance to the origin for the four quadrants. Some motion is observed in the middle of the domain. This problem is caused by the combination of wall heating, see Noh [37, and the presence of cells of different size in the middle of the domain. These heterogeneities produce a nonuniform density in the different quadrants which causes the more dense material to push the less dense one. One way to limit this effect consists of smoothing the initial length scale $h_{0}$. The initial length scale that is used in the computation is shown in the left panel of Figure 16. Although a large smoothing constant $\varepsilon=30.0$ has been used in the smoothing procedure (36), the large gradient on $h_{0}$ in the middle of the domain causes mesh deformation. Using a uniform initial length scale is not appropriate either, since it would cause adding too much (or too little) 

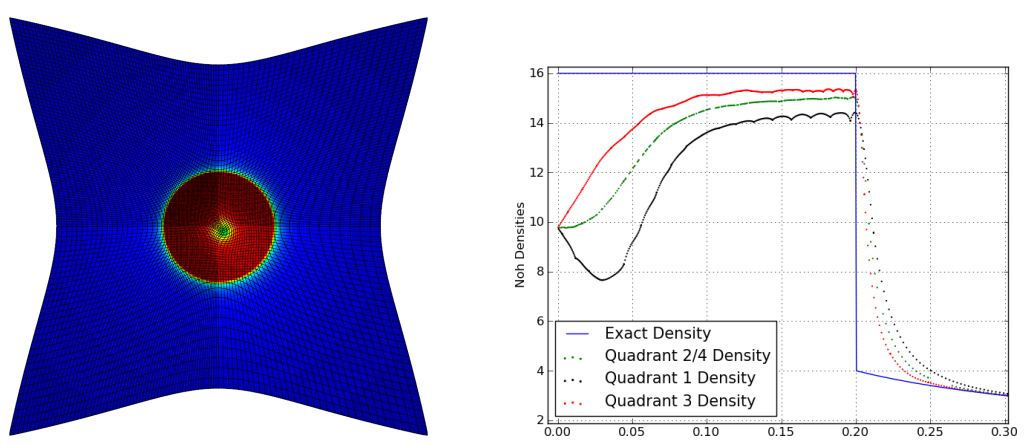

Figure 15: 2D Noh problem on a non-uniform mesh at $t=0.6$. Density and mesh (left panel), density vs. radius (right panel)

artificial viscosity to one of the quadrants. The right panel of Figure 16 shows the pressure field. The wall heating already mentioned above is clearly visible in the middle of the domain.
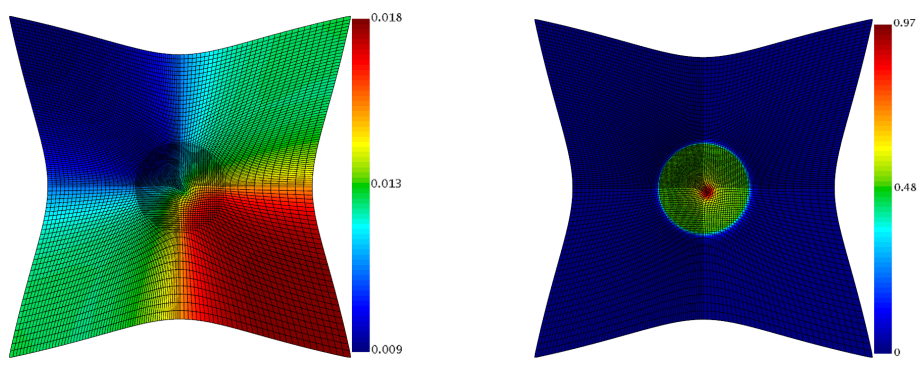

Figure 16: 2D Noh problem on a non-uniform mesh at $t=0.6$. Length scale field $h_{0}$ (left panel) and pressure (right panel).

\subsection{D Noh implosion and parallel performance}

Here we demonstrate the capabilities of the method to perform parallel 3D calculations. We consider the three-dimensional Noh problem with the initial conditions

$$
\rho\left(\boldsymbol{x}_{0}\right)=1, \quad \boldsymbol{u}\left(\boldsymbol{x}_{0}\right)=-\frac{\boldsymbol{r}}{\|\boldsymbol{r}\|_{\ell^{2}}}, e\left(\boldsymbol{x}_{0}\right)=0, \gamma=\frac{5}{3},
$$

Again we run this test up to $t=0.6$ with the entropy viscosity from Option 1 .

Although the purpose of the present paper is not to detail the computational aspects of the method; we have programmed the algorithm in parallel and in three space dimensions to give some ideas on the performances in realistic situations. Communication between parallel tasks occurs for the following procedures:

- Computation of the time step (47). 
- Computation of the global entropy production normalization constant used in the denominator of equation (44).

- Assembly of global matrices for density, velocity and specific internal energy at each Runge-Kutta sub-stage.

- Solving the mass matrix linear systems for density, velocity and specific internal energy, and redistribution of the new solution to the different MPI tasks at each Runge-Kutta sub-stage.

Figures 17 and 18 show the density and the mesh using $Q_{2}$ approximation on a uniform Cartesian mesh composed of 32 cells in each space direction. The initial domain is $\Omega_{0}=[-1.2,1.2]^{3}$. The center panel of Figure 18 shows the mesh division between 64 parallel tasks. We also present a strong scalability test for $Q_{2}$ (on $32^{3}$ cells) and $Q_{4}$ (on $16^{3}$ cells) simulations. We have performed 20 time steps for all the scalability simulations.
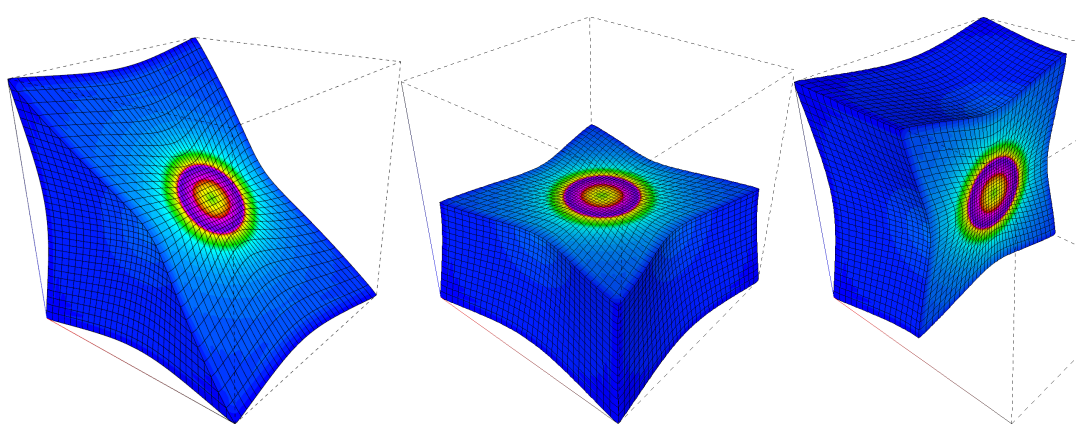

Figure 17: Density cuts for the 3D Noh problem at $t=0.6$.

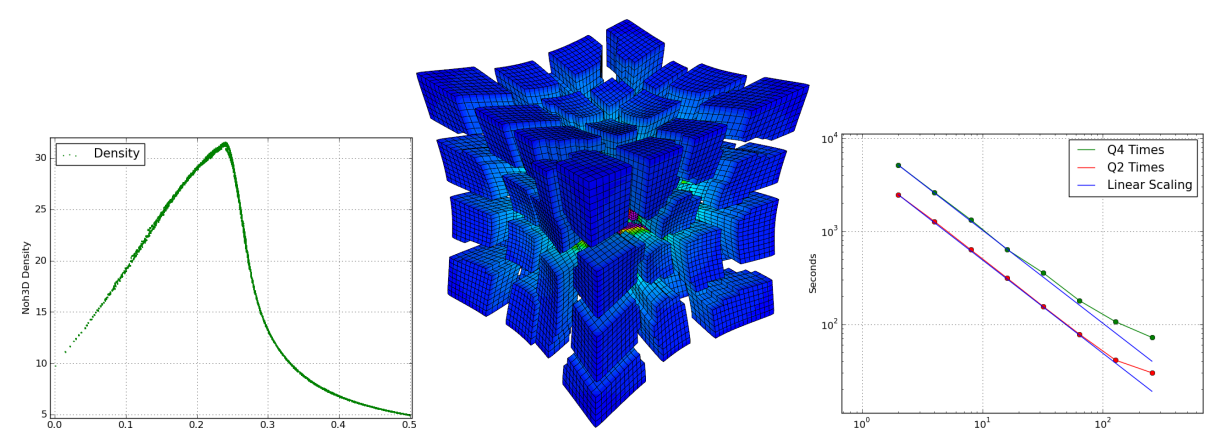

Figure 18: 3D Noh problem at $t=0.6$. Density vs. radius (left panel), 64 MPI tasks division (center panel), and strong scalability test from 1 to 256 processors (right panel). 


\section{Conclusion}

We have presented a new high-order curvilinear finite element method for Lagrangian hydrodynamics that is non-oscillatory in contacts, has a good shock detection capability, is compatible at the continuous level with generalized entropy inequalities $(15)$ and the minimum principle on the specific entropy (16). The artificial viscosity is also compatible with the requirements from Kolev and Rieben [26. The method is general with respect to the polynomial degree of the finite element approximation in space and the order of the time integration. The results reported confirm that the artificial viscosity converges to zero for smooth solutions and the convergence rate is optimal. The method achieves convergence with a rate close to one for standard shock wave problems, which is optimal. Proper radial symmetry preservation is observed on uniform and non-uniform meshes. The method has the ability to represent details of the flow within one single zone for high-order polynomial degrees. All these features come with the price of adding artificial viscosity in all the equations and using continuous finite element spaces for all variables. Being more diffusive, Option 1 is overall slightly more robust than Option 2. Our next objective is to replace the notion of artificial viscosity based on differential operators like $-\nabla \cdot(\nu \nabla)$ by a graph Laplacian approach outlined in http://arxiv.org/abs/1509.07461.

\section{Appendix}

\subsection{Orthogonal transformations invariance}

Here we show that the artificial viscosity is invariant under any orthogonal transformation of $\boldsymbol{x}$ and $\boldsymbol{u}$ as stated in Subsection 3.6 .

Proposition 1. The scalar diffusion in the mass equation (24) and the energy equation (26) are invariant by orthogonal transformation of $\boldsymbol{x}$ and $\boldsymbol{u}$. The viscous force in the momentum equation (25) is transformed the same way as $\boldsymbol{x}$ and $\boldsymbol{u}$.

Proof. Since this argument is discrete, we introduce the notation:

$$
\begin{aligned}
\rho(\boldsymbol{x}, t) & =\sum_{j=1}^{N} \rho_{j}(t) \varphi_{j}(\boldsymbol{x}, t), \quad e(\boldsymbol{x}, t)=\sum_{j=1}^{N} e_{j}(t) \varphi_{j}(\boldsymbol{x}, t), \\
u_{m}(\boldsymbol{x}, t) & =\sum_{j=1}^{N} U_{j m}(t) \varphi_{j}(\boldsymbol{x}, t), \quad m=1 \ldots d, \quad \boldsymbol{U}_{j}=\left(U_{j 1} \ldots U_{j d}\right)^{T}, \\
x_{m}\left(\boldsymbol{x}_{0}, t\right) & =\sum_{j=1}^{N} X_{j m}(t) \varphi_{j}\left(\boldsymbol{x}_{0}\right), \quad m=1 \ldots d, \quad \boldsymbol{X}_{j}=\left(X_{j 1} \ldots X_{j d}\right)^{T},
\end{aligned}
$$

where the details of the mesh representation can be found in 32 - 35 . Let $A$ be unitary matrix and consider the orthogonal transformation

$$
\boldsymbol{X}_{j}^{*}=A \boldsymbol{X}_{j}, \quad \boldsymbol{U}_{j}^{*}=A \boldsymbol{U}_{j}, \quad \text { where } A^{T}=A^{-1} .
$$


By convention all quantities used with upper index $*$ are defined in the transformed frame, i.e., are functions of $\boldsymbol{x}^{*}$. The Jacobian $J^{*}$ of the transformation $\widehat{\boldsymbol{x}} \rightarrow \boldsymbol{x}^{*}$ can be expressed by the Jacobian of $\widehat{\boldsymbol{x}} \rightarrow \boldsymbol{x} \rightarrow \boldsymbol{x}^{*}$ :

$$
J^{*}=A J, \quad \operatorname{det}\left(J^{*}\right)=\operatorname{det}(J) .
$$

Then the gradient of the finite element shape functions are obtained as follows:

$$
\nabla \varphi^{*}=\left(J^{*}\right)^{-T} \nabla \widehat{\varphi}=A J^{-T} \nabla \widehat{\varphi} .
$$

and the gradient of $\boldsymbol{u}^{*}$ is

$\nabla \boldsymbol{u}^{*}=\sum_{j=1}^{N} \boldsymbol{U}_{j}^{*} \otimes \nabla \varphi_{j}^{*}=\sum_{j=1}^{N}\left(A \boldsymbol{U}_{j}\right) \otimes\left(A J^{-T} \nabla \widehat{\varphi}_{j}\right)=\sum_{j=1}^{N} A \boldsymbol{U}_{j}\left(\nabla \varphi_{j}\right)^{T} A^{T}=A \nabla \boldsymbol{u} A^{T}$.

Lets examine the contribution of the artificial viscosity to a node $j$ in the mass equation (28). After mapping everything to the reference frame and applying a quadrature rule with weights and nodes $\left(w_{q}, \widehat{\boldsymbol{x}}_{q}\right)_{1 \leq q \leq Q}$, the contribution from an arbitrary cell $K^{*}$ is computed by

$$
-\sum_{q=1}^{Q}\left(w_{q}\left|\operatorname{det}\left(J_{q}^{*}\right)\right| \lambda_{q}^{*} \sum_{i=1}^{N} \rho_{i}\left(A J_{q}^{-T} \nabla \widehat{\varphi}_{i q}\right) \cdot\left(A J_{q}^{-T} \nabla \widehat{\varphi}_{j q}\right)\right),
$$

where $\nabla \widehat{\varphi}_{i q}:=\nabla \widehat{\varphi}_{i}\left(\widehat{\boldsymbol{x}}_{q}\right), J_{q}:=J\left(\widehat{\boldsymbol{x}}_{q}\right), \lambda_{q}^{*}:=\lambda^{*}\left(\boldsymbol{x}_{q}^{*}\right)$, etc. But this is the same as

$$
-\sum_{q=1}^{Q}\left(w_{q}\left|\operatorname{det}\left(J_{q}\right)\right| \lambda_{q}^{*} \sum_{i=1}^{N} \rho_{i}\left(J_{q}^{-T} \nabla \widehat{\varphi}_{i q}\right) \cdot\left(J^{-T} \nabla \widehat{\varphi}_{j q}\right)\right),
$$

which is the contribution from the corresponding cell $K$ if $\lambda^{*}\left(\boldsymbol{x}_{q}^{*}\right)=\lambda\left(\boldsymbol{x}_{q}\right)$. Now we look at the artificial force term in the momentum equation (29). The contribution of the Cartesian component $m$ from a cell $K^{*}$ to a node $j$ is

$$
\begin{aligned}
\sum_{q=1}^{Q} w_{q}\left|\operatorname{det}\left(J_{q}^{*}\right)\right|( & -\nu_{q}^{*} \widehat{\rho}_{q} \sum_{i=1}^{N} U_{i m}^{*}\left(A J_{q}^{-T} \nabla \widehat{\varphi}_{i q}\right) \cdot\left(A J_{q}^{-T} \nabla \widehat{\varphi}_{j q}\right) \\
& \left.+\lambda_{q}^{*} \widehat{\varphi}_{j q} \sum_{k=1}^{N} \sum_{i=1}^{N} U_{k m}^{*} \rho_{i}\left(A J_{q}^{-T} \nabla \varphi_{k q}\right) \cdot\left(A J^{-T} \nabla \varphi_{i q}\right)\right)= \\
\sum_{q=1}^{Q} w_{q}\left|\operatorname{det}\left(J_{q}\right)\right| & \left(-\nu_{q}^{*} \widehat{\rho}_{q} \sum_{i=1}^{N} A_{m} \cdot \boldsymbol{U}_{i}\left(J_{q}^{-T} \nabla \varphi_{i q}\right) \cdot\left(J_{q}^{-T} \nabla \varphi_{j q}\right)\right. \\
& \left.+\lambda_{q}^{*} \widehat{\varphi}_{j q} \sum_{k=1}^{N} \sum_{i=1}^{N} A_{m} \cdot \boldsymbol{U}_{k} \rho_{i}\left(J_{q}^{-T} \nabla \widehat{\varphi}_{k q}\right) \cdot\left(J_{q}^{-T} \nabla \widehat{\varphi}_{i q}\right)\right)
\end{aligned}
$$

where $A_{m}$ is the $m$-th row of A. Hence the artificial viscosity force is transformed the same way as $\boldsymbol{u}$ and $\boldsymbol{x}$ are if $\lambda^{*}\left(\boldsymbol{x}_{q}^{*}\right)=\lambda_{q}\left(\boldsymbol{x}_{q}\right)$ and $\nu^{*}\left(\boldsymbol{x}_{q}^{*}\right)=\nu\left(\boldsymbol{x}_{q}\right)$. The 
viscous terms in the internal energy equation (30) can be handled similarly and we reach the same conclusion. The only non trivial term there is $\left\|\nabla \boldsymbol{u}^{*}\right\|_{\ell^{2}}^{2}$; but we have $\nabla \boldsymbol{u}^{*}: \nabla \boldsymbol{u}^{*}=\nabla \boldsymbol{u}: \nabla \boldsymbol{u}$ since the Frobenius norm is invariant under orthogonal transformations. Hence the orthogonal transformation invariance holds if $\lambda_{q}^{*}=\lambda_{q}$ and $\nu_{q}^{*}=\nu_{q}$.

Now we consider the shock detectors. For the entropy production 42 we have $D^{*}=D$ since it depends only on scalars and $\nabla \cdot \boldsymbol{u}^{*}$ :

$$
\begin{aligned}
\nabla \cdot \boldsymbol{u}^{*} & =\sum_{j=1}^{N} \nabla \varphi_{j}^{*} \cdot \boldsymbol{U}_{j}^{*}=\sum_{j=1}^{N}\left(A J^{-T} \nabla \widehat{\varphi}_{j}\right) \cdot\left(A \boldsymbol{U}_{j}\right)= \\
& =\sum_{j=1}^{N}\left(A \nabla \varphi_{j}\right) \cdot\left(A \boldsymbol{U}_{j}\right)=\sum_{j=1}^{N} \nabla \varphi_{j} \cdot \boldsymbol{U}_{j}=\nabla \cdot \boldsymbol{u} .
\end{aligned}
$$

The first-order viscosity of Option 1 in 40 is also invariant since $\left\|\boldsymbol{u}^{*}\right\|_{\ell^{2}}=$ $\|A \boldsymbol{u}\|_{\ell^{2}}=\|\boldsymbol{u}\|_{\ell^{2}}$. For Option 2, see (41), we have $\nabla^{s} \boldsymbol{u}^{*}=A \nabla^{s} \boldsymbol{u} A^{T}$, hence $\nabla^{s} \boldsymbol{u}^{*}$ and $\nabla^{s} \boldsymbol{u}$ are similar matrices; thereby implying that $\mu^{*}=\mu$ and $\boldsymbol{s}^{*}=A s$, where recall that $(\mu, s)$ is an eigenpair of $\nabla^{s} \boldsymbol{u}$. Hence the first-order viscosity is invariant under orthogonal transformation in both Option 1 and Option 2.

We now investigate how the length scales are transformed. It is clear that $h_{0}^{*}=h_{0}$ since lengths and angles are invariant by orthogonal transformation. Consider the Jacobian of the transformation $\boldsymbol{x}_{0}^{*} \rightarrow \boldsymbol{x}^{*}$, that is to say, the Jacobian of $\boldsymbol{x}_{0}^{*} \rightarrow \boldsymbol{x}_{0} \rightarrow \boldsymbol{x} \rightarrow \boldsymbol{x}^{*}$, which is $A J J_{0}^{-1} A^{T}$, then we have

$$
\begin{gathered}
\operatorname{det}(A)=1 \Rightarrow \operatorname{det}\left(A J J_{0}^{-1} A^{T}\right)=\operatorname{det}\left(J J_{0}^{-1}\right) \Rightarrow h_{3}^{*}=h_{3} . \\
\frac{\left\|A J J_{0}^{-1} A^{T} \boldsymbol{u}^{*}\right\|_{\ell^{2}}}{\left\|\boldsymbol{u}^{*}\right\|_{\ell^{2}}}=\frac{\left\|A J J_{0}^{-1} \boldsymbol{u}\right\|_{\ell^{2}}}{\|A \boldsymbol{u}\|_{\ell^{2}}}=\frac{\left\|J J_{0}^{-1} \boldsymbol{u}\right\|_{\ell^{2}}}{\|\boldsymbol{u}\|_{\ell^{2}}} \Rightarrow h_{1}^{*}=h_{1} . \\
\frac{\left\|A J J_{0}^{-1} A^{T} \boldsymbol{s}^{*}\right\|_{\ell^{2}}}{\left\|\boldsymbol{s}^{*}\right\|_{\ell^{2}}}=\frac{\left\|A J J_{0}^{-1} \boldsymbol{s}\right\|_{\ell^{2}}}{\|A \boldsymbol{s}\|_{\ell^{2}}}=\frac{\left\|J J_{0}^{-1} \boldsymbol{s}\right\|_{\ell^{2}}}{\|\boldsymbol{s}\|_{\ell^{2}}} \Rightarrow h_{2}^{*}=h_{2} .
\end{gathered}
$$

This implies that $\lambda^{*}=\lambda$ and $\nu^{*}=\nu$ for all the options.

\subsection{Parameter list}

In this section we list all the parameters that have been used in the computations reported in the paper. Are listed the viscosity options, the polynomial degree of the finite element space, the size of the initial mesh, the Runge-Kutta

method, the CFL constant $c_{\mathrm{cfl}}$, the viscosity constants $c_{\mathrm{visc}}, c_{\mathrm{entr}}$, and the final time $t$.

- 2D Taylor-Green problem, all figures $\left(c_{\mathrm{cfl}}=0.5\right)$ and convergence tables $\left(c_{\mathrm{cfl}}=0.1\right)$ : Option 2 (with $h_{3}$ on $\left.|D|\right), \mathrm{RK} 4, c_{\mathrm{visc}}=1.0, c_{\mathrm{entr}}=0.5, t=$ 0.5 .

- 1D Sod tube, Figure 6 Option 2 (with $h_{3}$ on $|D|$ ), $Q_{1}, 128$ cells, RK4, $c_{\mathrm{cfl}}=0.2, c_{\mathrm{visc}}=1.0, t=0.2$. 
- 1D Sod tube, Table 3, Figure 7, Figure 8 Option 1 (with $h_{3}$ on $|D|$ ), 256 cells for $Q_{4}, 1024$ cells for $Q_{1}, \mathrm{RK} 4, c_{\mathrm{cfl}}=0.4, c_{\mathrm{visc}}=0.5, c_{\mathrm{entr}}=20.0, t=$ 0.2 .

- 1D Sod tube, Figure 8, $1024 Q_{1}$ cells with the same parameters as above.

- 2D Sedov explosion, Figure 9, Figure 11. Option 2 (with $h_{3}$ on $|D|$ ), $Q_{2}$, $\mathrm{RK} 3,64 \times 64, c_{\mathrm{cfl}}=0.2, c_{\mathrm{visc}}=2.0, c_{\mathrm{entr}}=5.0, t=1.0$.

- 2D Sedov explosion, Figure 12 Option 2 (with $h_{3}$ on $|D|$ ), $Q_{2}$, RK3, $64 \times 64$ cells in quadrant \#2, $32 \times 64$ in \#1, $64 \times 32$ in \#3, $32 \times 32$ in \#4, $c_{\mathrm{cfl}}=0.15, c_{\mathrm{visc}}=2.0, c_{\mathrm{entr}}=20.0, t=1.0, \varepsilon=1.0$ for $h_{0}$ smoothing.

- 2D Noh implosion, Figure 13, Figure 14 Option 1 (with $h_{1}$ on $|D|$ ), $Q_{2}$, $\mathrm{RK} 4,64 \times 64, c_{\mathrm{cfl}}=0.05, c_{\mathrm{visc}}=0.75, c_{\mathrm{entr}}=20.0, t=0.6$.

- 2D Noh implosion, Figure 15, Figure 16 Option 1 (with $h_{1}$ on $|D|$ ), $Q_{2}$, RK4, $64 \times 64$ cells in quadrant $\# 2,32 \times 64$ in \#1, $64 \times 32$ in \#3, $32 \times 32$ in $\# 4, c_{\mathrm{cfl}}=0.05, c_{\mathrm{visc}}=0.75, c_{\mathrm{entr}}=20.0, t=0.6, \varepsilon=30.0$ for $h_{0}$ smoothing.

- 3D Noh implosion, Figure 17, Figure 18 Option 1 (with $h_{1}$ on $\left.|D|\right), Q_{2}$ $/ Q_{4}, R K 4,32 \times 32 \times 32 / 16 \times 16 \times 16, c_{\mathrm{cfl}}=0.05, c_{\mathrm{visc}}=0.75, c_{\mathrm{entr}}=$ $20.0, t=0.6$.

\section{References}

[1] A. Barlow. A compatible finite element multi-material ALE hydrodynamics algorithm. Internat. J. Numer. Methods Fluids, 56:953-964, 2007.

[2] Y. Bazilevs, I. Akkerman, D. Benson, G. Scovazzi, and M. Shashkov. Isogeometric analysis of lagrangian hydrodynamics. Journal of Computational Physics, 243(0):224 - 243, 2013.

[3] D. J. Benson. Computational methods in Lagrangian and Eulerian hydrocodes. Comput. Methods Appl. Mech. Engrg., 99:235-394, 1992.

[4] S. Bianchini and A. Bressan. Vanishing viscosity solutions of nonlinear hyperbolic systems. Ann. of Math. (2), 161(1):223-342, 2005.

[5] D. Burton. Exact conservation of energy and momentum in staggeredgrid hydrodynamics with arbitrary connectivity. In Advances in the FreeLagrange Method Including Contributions on Adaptive Gridding and the Smooth Particle Hydrodynamics Method, volume 395, pages 7-19. Springer Berlin Heidelberg, 1991.

[6] D. E. Burton. Multidimensional discretizations of conservation laws for unstructured polyhedral grids. Technical Report UCRL-JC-118306, Lawrence Livermore National Laboratory, 1994. 
[7] E. Caramana, D. Burton, and M. Shashkov. The construction of compatible hydrodynamics algorithms utilizing conservation of total energy. $J$. Comput. Phys., 146(1):227-262, 1998.

[8] E. Caramana, M. Shashkov, and P. Whalen. Formulations of artificial viscosity for multi-dimensional shock wave computations. J. Comput. Phys., 144(1):70-97, 1998.

[9] E. J. Caramana and R. Loubère. "Curl-q": a vorticity damping artificial viscosity for essentially irrotational Lagrangian hydrodynamics calculations. J. Comput. Phys., 215(2):385-391, 2006.

[10] G. Carré, S. Del Pino, B. Després, and E. Labourasse. A cell-centered lagrangian hydrodynamics scheme on general unstructured meshes in arbitrary dimension. J. Comput. Phys., 228(14):5160-5183, 2009.

[11] A. Claisse, B. Desprs, E. Labourasse, and F. Ledoux. A new exceptional points method with application to cell-centered lagrangian schemes and curved meshes. Journal of Computational Physics, 231(11):4324 - 4354, 2012 .

[12] B. Després and C. Mazeran. Lagrangian gas dynamics in two dimensions and Lagrangian systems. Arch. Rational Mech. Anal., 178(3):327-372, 2005.

[13] X. Ding, G. Chen, and P. Luo. Convergence of the Lax-Friedrichs scheme for isentropic gas dynamics I. Acta. Math. Sci., 5:415-432, 1985.

[14] R. J. DiPerna. Convergence of approximate solutions to conservation laws. Arch. Rational Mech. Anal., 82(1):27-70, 1983.

[15] R. J. DiPerna. Convergence of the viscosity method for isentropic gas dynamics. Comm. Math. Phys., 91(1):1-30, 1983.

[16] V. Dobrev, T. Kolev, and R. Rieben. High-order curvilinear finite element methods for Lagrangian hydrodynamics. SIAM J. Sci. Comput., 34(5): B606-B641, 2012.

[17] H. Frid. Maps of convex sets and invariant regions for finite-difference systems of conservation laws. Arch. Ration. Mech. Anal., 160(3):245-269, 2001.

[18] GLVis. OpenGL visualization tool. URL http://glvis.googlecode.com

[19] J.-L. Guermond. On the use of the notion of suitable weak solutions in CFD. Internat. J. Numer. Methods Fluids, 57(9):1153-1170, 2008.

[20] J.-L. Guermond and B. Popov. Viscous regularization of the euler equations and entropy principles. SIAM Journal on Applied Mathematics, 74(2):284$305,2014$. 
[21] J.-L. Guermond and B. Popov. Invariant domains and first-order continuous finite element approximation for hyperbolic systems. SIAM Journal on Numerical Analysis, 2015.

[22] J.-L. Guermond, M. Nazarov, and B. Popov. Implementation of the entropy viscosity method. Technical Report 4015, KTH, Numerical Analysis, Sweden, Stockholm, 2011. QC 20110720.

[23] J.-L. Guermond, R. Pasquetti, and B. Popov. Entropy viscosity method for nonlinear conservation laws. J. Comput. Phys., 230(11):4248-4267, 2011.

[24] A. Harten. On the symmetric form of systems of conservation laws with entropy. J. Comput. Phys., 49(1):151-164, 1983.

[25] A. Harten, P. Lax, D. Levermore, and W. Morokoff. Convex entropies and hyperbolicity for general Euler equations. SIAM J. Numer. Anal., 35(6): 2117-2127, 1998.

[26] T. Kolev and R. Rieben. A tensor artificial viscosity using a finite element approach. J. Comput. Phys., 228(22):8336-8366, 2009.

[27] S. Krushkov. First order quasilinear equations with several independent variables. Math. USSR-Sb., 10:217-243, 1970.

[28] P. Lax. Weak solutions of nonlinear hyperbolic equations and their numerical computation. Comm. Pure Appl. Math., 7:159-193, 1954.

[29] P. Lax. Shock Waves and Entropy in Contributions to Nonlinear Functional Analysis. Academic Press, New York, NY, 1971.

[30] K. Lipnikov and M. Shashkov. A framework for developing a mimetic tensor artificial viscosity for Lagrangian hydrocodes on arbitrary polygonal meshes. J. Comput. Phys., 229(20):7911-7941, 2010.

[31] R. Loubère, P.-H. Maire, and P. Váchal. 3d staggered lagrangian hydrodynamics scheme with cell-centered riemann solver-based artificial viscosity. International Journal for Numerical Methods in Fluids, 72(1):22-42, 2013.

[32] P.-H. Maire. A high-order cell-centered Lagrangian scheme for twodimensional compressible fluid flows on unstructured meshes. J. Comput. Phys., 228(7):2391-2425, 2009.

[33] P.-H. Maire and J. Breil. A second-order cell-centered lagrangian scheme for two-dimensional compressible flow problems. Internat. J. Numer. Methods Fluids, 56(8):1417-1423, 2008.

[34] P.-H. Maire, R. Abgrall, J. Breil, and J. Ovadia. A cell-centered Lagrangian scheme for two-dimensional compressible flow problems. SIAM J. Sci. Comp., 29(4):1781-1824, 2007. 
[35] P.-H. Maire, R. Loubère, and P. Vachal. Staggered Lagrangian discretization based on cell-centered Riemann solver and associated hydrodynamics scheme. Commun. Comput. Phys., 10:940-978, 2011.

[36] MFEM. Modular finite element methods library. URL http://mfem. googlecode.com.

[37] W. Noh. Errors for calculations of strong shocks using an artificial viscosity and an artificial heat flux. J. Comput. Phys., 72(1):78-120, 1987.

[38] G. Scovazzi. Stabilized shock hydrodynamics: II. Design and physical interpretation of the SUPG operator for Lagrangian computations. Comput. Methods Appl. Mech. Engrg., 196:966-978, 2007.

[39] G. Scovazzi, E. Love, and M. Shashkov. Multi-scale Lagrangian shock hydrodynamics on $\mathrm{Q} 1 / \mathrm{P} 0$ finite elements: Theoretical framework and twodimensional computations. Comput. Methods Appl. Mech. Engrg., 197: 1056-1079, 2008.

[40] L. Sedov. Similarity and dimensional methods in mechanics. CRC Press, Boca Raton, FL, 1993.

[41] D. Serre. Systèmes de lois de conservation I: hyperbolicité, entropies, ondes de choc. Diderot Editeur, Paris, 1996.

[42] M. Shashkov and J. Campbell. A tensor artificial viscosity using a mimetic finite difference algorithm. J. Comput. Phys., 172(2):739-765, 2001.

[43] E. Tadmor. A minimum entropy principle in the gas dynamics equations. Appl. Numer. Math., 2(3-5):211-219, 1986.

[44] F. Vilar, P.-H. Maire, and R. Abgrall. A discontinuous galerkin discretization for solving the two-dimensional gas dynamics equations written under total lagrangian formulation on general unstructured grids. J. Comput. Phys., 276(0):188 - 234, 2014.

[45] J. Von Neumann and R. Richtmyer. A method for the numerical calculation of hydrodynamic shocks. J. Appl. Phys., 21:232-237, 1950.

[46] M. L. Wilkins. Methods in Computational Physics, volume 3. Academic Press, 1964.

[47] V. Zingan, J.-L. Guermond, J. Morel, and B. Popov. Implementation of the entropy viscosity method with the discontinuous Galerkin method. Comput. Methods Appl. Mech. Engrg., 253:479-490, 2013. 